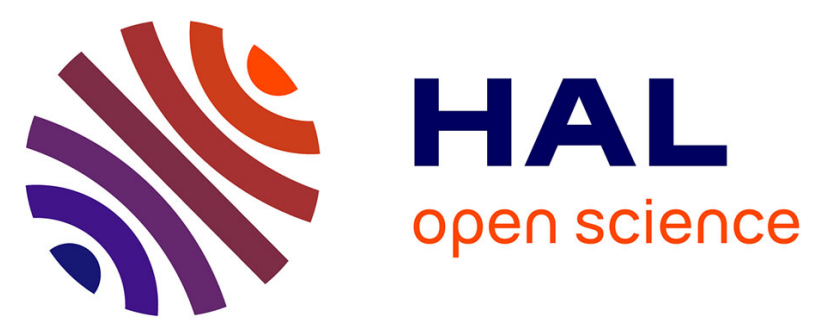

\title{
Functional diversification in the Nudix hydrolase gene family drives sesquiterpene biosynthesis in Rosa $\mathrm{x}$ wichurana
}

\author{
Pulu Sun, Clément Dégut, Stéphane Réty, Jean-Claude Caissard, Laurence \\ Hibrand-Saint Oyant, Raymonde Baltenweck, Aurélie Bony, Saretta N \\ Paramita, Corentin Conart, Jean-louis Magnard, et al.
}

\section{To cite this version:}

Pulu Sun, Clément Dégut, Stéphane Réty, Jean-Claude Caissard, Laurence Hibrand-Saint Oyant, et al.. Functional diversification in the Nudix hydrolase gene family drives sesquiterpene biosynthesis in Rosa x wichurana. Plant Journal, 2020, 104 (1), pp.185-199. 10.1111/tpj.14916 . hal-02990985

\section{HAL Id: hal-02990985 \\ https://hal.science/hal-02990985}

Submitted on 13 Nov 2020

HAL is a multi-disciplinary open access archive for the deposit and dissemination of scientific research documents, whether they are published or not. The documents may come from teaching and research institutions in France or abroad, or from public or private research centers.
L'archive ouverte pluridisciplinaire HAL, est destinée au dépôt et à la diffusion de documents scientifiques de niveau recherche, publiés ou non, émanant des établissements d'enseignement et de recherche français ou étrangers, des laboratoires publics ou privés. 


\title{
Functional diversification in the Nudix hydrolase gene family drives sesquiterpene biosynthesis in Rosa $\times$ wichurana
}

Pulu Sun $^{1,2}$ iD, Clément Dégut ${ }^{3}$ (iD), Stéphane Réty ${ }^{4}$ (iD, Jean-Claude Caissard ${ }^{1}$ (iD, Laurence Hibrand-Saint Oyant ${ }^{5}$ (iD, Aurélie Bony ${ }^{1}$, Saretta N. Paramita ${ }^{1}$, Corentin Conart' ${ }^{1}$ Jean-Louis Magnard ${ }^{1}$, Julien Jeauffre ${ }^{5}$, Ahmed M. Abd-El-Haliem ${ }^{2}$ iD, Jordan Marie-Magdelaine ${ }^{5}$, Tatiana Thouroude ${ }^{5}$, Raymonde Baltenweck ${ }^{6}$, Carine Tisné ${ }^{3}$ (iD, Fabrice Foucher $^{5}$ (D), Michel Haring ${ }^{2}$, Philippe Hugueney ${ }^{6}$ (D), Robert C. Schuurink ${ }^{2}$ (D) and Sylvie Baudino ${ }^{1 *}$

${ }^{1}$ Univ Lyon, UJM-Saint-Etienne, CNRS, BVpam FRE 3727, Saint-Etienne F-42023, France,

${ }^{2}$ Green Life Sciences Research Cluster, Swammerdam Institute for Life Sciences, University of Amsterdam, Science Park 904, Amsterdam $1098 \mathrm{XH}$, The Netherlands,

${ }^{3}$ Expression Génétique Microbienne, UMR 8261, CNRS, Université de Paris, Institut de Biologie Physico-Chimique (IBPC), Paris 75005, France,

${ }^{4}$ Univ Lyon, ENS de Lyon, Univ Claude Bernard, CNRS UMR 5239, INSERM U1210, LBMC, 46 Allée d'Italie Site Jacques Monod, Lyon F-69007, France,

5IRHS-UMR1345, Université d'Angers, INRAE, Institut Agro, SFR 4207 QuaSaV, Beaucouzé 49071, France, and

${ }^{6}$ Université de Strasbourg, INRAE, SVOV UMR-A 1131, Colmar F-68000, France

Received 23 April 2020; revised 3 June 2020; accepted 24 June 2020; published online 8 July 2020.

*For correspondence (e-mail sylvie.baudino@univ-st-etienne.fr).

\begin{abstract}
SUMMARY
Roses use a non-canonical pathway involving a Nudix hydrolase, RhNUDX1, to synthesize their monoterpenes, especially geraniol. Here we report the characterization of another expressed NUDX1 gene from the rose cultivar Rosa x wichurana, RwNUDX1-2. In order to study the function of the RwNUDX1-2 protein, we analyzed the volatile profiles of an $\mathrm{F}_{1}$ progeny generated by crossing $R$. chinensis cv. 'Old Blush' with $R . x$ wichurana. A correlation test of the volatilomes with gene expression data revealed that RwNUDX1-2 is involved in the biosynthesis of a group of sesquiterpenoids, especially $E, E$-farnesol, in addition to other sesquiterpenes. In vitro enzyme assays and heterologous in planta functional characterization of the RwNUDX1-2 gene corroborated this result. A quantitative trait locus (QTL) analysis was performed using the data of $E, E$-farnesol contents in the progeny and a genetic map was constructed based on gene markers. The RwNUDX1-2 gene co-localized with the OTL for E,E-farnesol content, thereby confirming its function in sesquiterpenoid biosynthesis in $R . \times$ wichurana. Finally, in order to understand the structural bases for the substrate specificity of rose NUDX proteins, the RhNUDX1 protein was crystallized, and its structure was refined to $1.7 \AA$. By molecular modeling of different rose NUDX1 protein complexes with their respective substrates, a structural basis for substrate discrimination by rose NUDX1 proteins is proposed.
\end{abstract}

Keywords: Nudix hydrolase, rose scent, sesquiterpenes, farnesol, volatile compounds, quantitative trait locus, Nudix structure.

\section{INTRODUCTION}

Rose is one of the most economically important flowers, with thousands of cultivars mainly used as cut flowers, as garden ornamentals, and for the perfume industry. Fragrance is a very important rose trait that contributes to its commercial value, beside flower shape and petal color (Smulders et al., 2019). Hundreds of rose volatile compounds have been identified so far, including terpenoids, phenylpropanoids, and lipid-derived volatiles (Shalit et al., 2004). Many of the rose volatiles are commonly used in the perfume and cosmetic industry (Schwab et al., 2008). For example, geraniol, one of the major monoterpene alcohols $\left(\mathrm{C}_{10}\right.$ terpenoid) from rose petals, is responsible for their sweet floral rose smell (Chen and Viljoen, 2010). In many plant species, monoterpene biosynthesis relies on plastidlocalized terpene synthases (TPSs) such as geraniol synthase (GES) (lijima et al., 2004; Yang et al., 2005; Ito and Honda, 2007; Masumoto et al., 2010; Dong et al., 2013; Simkin et al., 2013). However, no TPS with GES activity has been characterized in roses to date. Instead, rose flowers 
use an alternative TPS-independent pathway to produce geraniol, involving a diphosphohydrolase belonging to the Nudix enzyme family. The cytosolic Nudix hydrolase RhNUDX1 converts geranyl diphosphate into geranyl monophosphate, which is then hydrolyzed to geraniol by petal-derived phosphatase activity (Magnard et al., 2015).

Nudix hydrolases have been identified in many species, including archaea, bacteria, eukaryotes, and viruses (Gunawardana et al., 2009). They were originally defined as housecleaning enzymes, eliminating toxic metabolites from the cells (Bessman et al., 1996). Nudix hydrolases constitute a superfamily of pyrophosphatases catalyzing the hydrolysis of nucleoside diphosphates linked to different X moieties (Bessman et al., 1996). Known substrates of Nudix hydrolases include nucleoside triphosphates (dNTPs) and their oxidized derivatives, such as 7,8-dihydro-8-oxo-deoxyguanosine triphosphate (8-oxo-dGTP), nucleotide sugars and alcohols, dinucleoside polyphosphates, dinucleotide coenzymes, and capped mRNAs (McLennan, 2006). Members of the Nudix hydrolase superfamily all share a conserved Nudix box. This Nudix motif is formed by a loop- $\alpha$ helix-loop structure, and provides binding sites for divalent cations (usually $\mathrm{Mg}^{2+}$ or $\mathrm{Mn}^{2+}$ ) that play a crucial role in catalysis. The presence of these cations is required for the activity of the Nudix proteins. The substrate specificity and the catalytic reaction mechanism are also determined by regions outside of the Nudix motif (McLennan, 2006; Gunawardana et al., 2009). For example, enzymes acting on Coenzyme $A$ share a motif that is located outside of the Nudix box and is involved in substrate recognition (Kupke et al., 2009). These regions, together with the Nudix motif, form an $\alpha / \beta / \alpha$ sandwich structure, which is also known as the Nudix fold (Mildvan et al., 2005). This fold is shared by the isopentenyl diphosphate isomerases, which, together with Nudix hydrolases and other proteins, form a larger group. This group was previously called the Nudix suprafamily (McLennan, 2006), but is sometimes also referred to as the Nudix superfamily or the Nudix homology clan (Srouji et al., 2017).

The functions of most Nudix hydrolases remain unclear in plants. For example, Nudix hydrolase 1 from Arabidopsis thaliana (AtNUDX1) was first described as an NADH pyrophosphatase (Dobrzanska et al., 2002). Under specific physiological conditions, AtNUDX1 was later shown to be involved in folate biosynthesis, using dihydroneopterin triphosphate as a substrate (Klaus et al., 2005). AtNUDX1 was also proposed to be involved in the elimination of harmful nucleoside- and deoxynucleoside-triphosphate derivatives such as 8-oxo-dGTP, similar to the MutT protein from Escherichia coli (Ogawa et al., 2005; Yoshimura et al., 2007). Recently, AtNUDX1 was shown to play a role in the regulation of terpene biosynthesis, by acting on isopentenyl diphosphate (IPP) precursors (Henry et al., 2018).
The rose Nudix hydrolase RhNUDX1 has been shown to be involved in the biosynthesis of the monoterpene geraniol, which is a major petal volatile in many scented roses (Magnard et al., 2015). However, besides monoterpenols, the petals of some rose species, such as Rosa x wichurana, emit significant amounts of sesquiterpenes (Roccia et al., 2019). Using a combination of molecular, genetic, and structural approaches, we characterized a novel NUDX1 protein from $R . \quad x$ wichurana, RwNUDX1-2. Unlike RhNUDX1, which is involved in monoterpene biosynthesis, RwNUDX1-2 uses farnesyl diphosphate (FPP) to produce farnesyl monophosphate (FP) as a precursor of $E, E$-farnesol and other sesquiterpenoids. Furthermore, we solved the crystal structure of the RhNUDX1 protein and used molecular modeling to provide structural bases for explaining the different substrate specificities of NUDX1 enzymes from different rose cultivars and from $A$. thaliana.

\section{RESULTS}

\section{NUDX1 genes in rose belong to a complex gene family organized in three clades: NUDX1-1 to NUDX1-3}

In order to further investigate the functions of Nudix hydrolases 1 in roses, we generated RNA-seq reads from four different rose cultivars: $R$. chinensis cv. 'Old Blush' $(\mathrm{OB}), R$. x wichurana (Rw), and two individuals (OW9035 and OW9047) obtained from a crossing between OB and Rw. Next, we pooled the generated reads together, used them to assemble a transcriptome de novo, and searched it to identify independent NUDX1 mRNA transcripts. Subsequently, we used the generated transcriptome as a reference to map the RNA-seq reads from each sample and to quantify gene expression. Four sequences were retrieved that were annotated as NUDX1 (Table S1). Among these sequences, one corresponded to the sequence previously characterized from $R$. x hybrida cv. 'Papa Meilland' (PM, RhNUDX1 in Magnard et al., 2015) and was highly expressed in the rose petals from OB (fragments per kilobase of transcript per million fragments mapped reads [FPKM]: 9347.2) and OW9047 (FPKM: 6687.3). We named it NUDX1-1. Another sequence was highly expressed in $\mathrm{Rw}$ (FPKM: 1433.0) and OW9035 (FPKM: 1332.6), but differed from the first sequence and was named NUDX1-2. It was noticeable that NUDX1-1 and NUDX1-2 were not highly expressed together in these four cultivars (Table S1). Two other sequences were partial and corresponded to another NUDX1 (NUDX1-3) but were weakly expressed in the rose petals of the four individuals, as judged by the read counts in the RNA-seq data. A search in the recently published genomes was used to retrieve all NUDX1 sequences in $\mathrm{OB}$ (Hibrand Saint-Oyant et al., 2018; Raymond et al., 2018). NUDX1-1 was present in five highly similar copies on chromosome 2 of the homozygous genomes (collectively named RcNUDX1-1a, see Figure S1) and one less similar 
copy on chromosome 4 (RcNUDX1-1b). NUDX1-2 was present in two copies in the homozygous genomes (RcNUDX1-2a and RcNUDX1-2b). A search in the heterozygous genome allowed us to detect a third copy (RcNUDX1$2 c$ ), missing in both homozygous genomes. NUDX1-3 was present in one copy in the homozygous genomes (RcNUDX1-3). Using genomic DNA from Rw as template, several corresponding sequences were amplified and named RwNUDX1-1, RwNUDX1-2a, RwNUDX1-2b, RwNUDX1-2c, RwNUDX1-2c', and RwNUDX1-3 (see Table S2 for primers). All NUDX1 genes were predicted to encode proteins of around 150 amino acids that contained the characteristic Nudix box (Figure 1a) (Bessman et al., 1996). Percentages of identity of these proteins are presented in Table S3. RwNUDX1-1 was identical to RcNUDX1-1b, RwNUDX1-3 was identical to RcNUDX1-3,

(a)

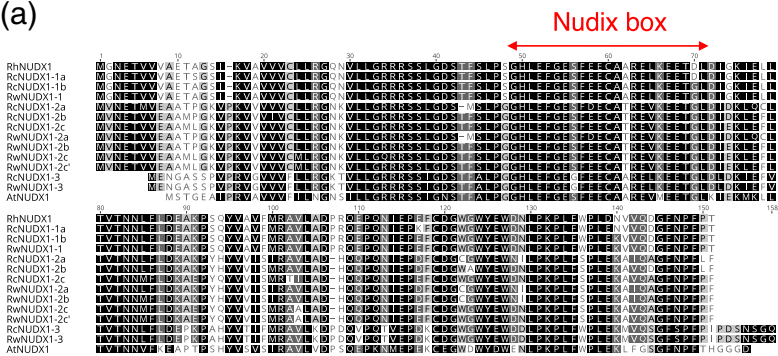

(b)

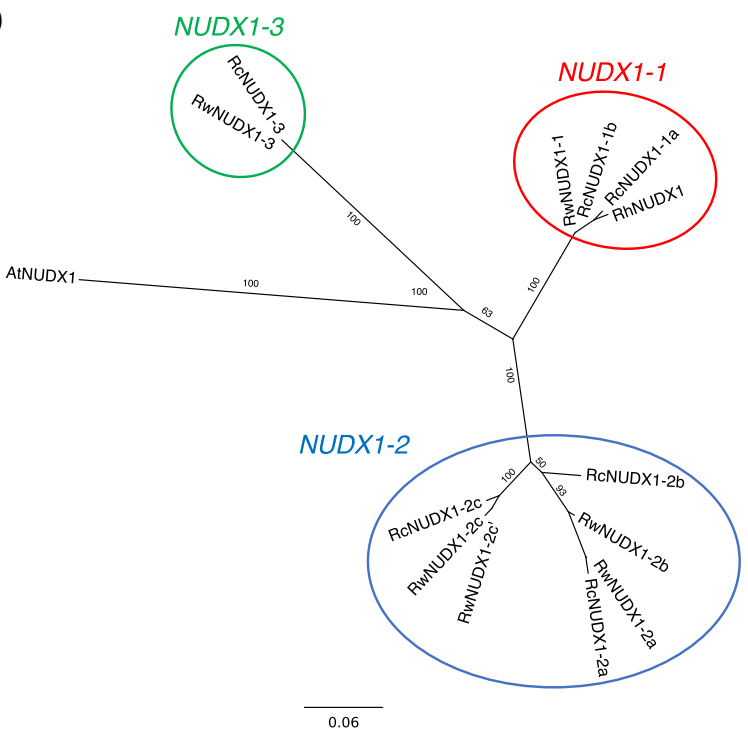

Figure 1. (a) Alignment of amino acid sequences of $R . x$ hybrida cv. 'Papa Meilland' (RhNUDX1), R. chinensis cv. 'Old Blush' (RcNUDX1-1a, RcNUDX11b, RcNUDX1-2a, RcNUDX1-2b, RcNUDX1-2c, and RcNUDX1-3), R. x wichurana (RwNUDX1-1, RwNUDX1-2a, RwNUDX1-2b, RwNUDX1-2c, RwNUDX1$2 c^{\prime}$, and RwNUDX1-3), and A. thaliana (AtNUDX1). The Nudix box corresponding to the consensus sequence Gx5Ex7REUxEExGU ( $x$, any amino acid; U, bulky hydrophobic amino acid, normally lle, Leu, or Val) is indicated. (b) Unrooted neighbor joining tree displaying the relationships of rose NUDX1 proteins with AtNUDX1 from $A$. thaliana (AtNUDX1). Alignment and tree were constructed using Geneious ${ }^{\circledR}$ software (version 10.2.3, Biomatters, Auckland, New Zealand), and the bootstrap values are shown as a percentage from 1000 bootstraps replicates. and RwNUDX1-2c and c' were very similar to RcNUDX1-2c. Alignment of rose NUDX1 protein sequences with AtNUDX1 and building of a phylogenetic tree confirmed that the sequences could be separated into three well-supported clades, named NUDX1-1, NUDX1-2, and NUDX1-3 (Figure 1b). RhNUDX1, RcNUDX1-1a, RcNUDX1-1b, and RwNUDX1-1 are closely grouped together, as are RcNUDX1-2a, b, and $c$ and RwNUDX1-2a, b, $c$, and $c$ '. RcNUDX1-3 and RwNUDX1-3 are in another cluster, to which AtNUDX1 is the closest. As NUDX1-1 and NUDX1-2 were highly expressed in petals (Table S1), we decided to focus on these two NUDX1 sequences.

NUDX1-1a expression is correlated with the production of geraniol and other monoterpenoids while NUDX1-2 expression is correlated with the production of $E, E$ farnesol and other sesquiterpenoid compounds

$\mathrm{OB}$ and Rw have distinct scent profiles: at the open flower stage (stage four according to Bergougnoux et al., 2007), OB produced mainly 1,3,5-trimethoxybenzene, geraniol, dihydro- $\beta$-ionol, and germacrene $D$, while $R w$ was rich in 2-phenylethanol (2PE) and $E$,E-farnesol (Table 1). In order to study the potential functions of NUDX1 genes, the expressions of NUDX1-1 and NUDX1-2 were analyzed in $\mathrm{OB}$ and $\mathrm{Rw}$ and in an $\mathrm{F}_{1}$ progeny from crosses between $\mathrm{OB}$ and Rw genotypes. This mapping population (OW) consists of a full-sib family of 151 hybrids (Hibrand SaintOyant et al., 2018). A small subset of this rose population, 18 out of 151 rose plants, including the parents $O B$ and $R w$, was subjected to quantitative reverse transcriptasePCR (qRT-PCR) to determine the transcript levels of NUDX1-1 and NUDX1-2 across the OW population at stage 4. It was already known that $R h N U D X 1$ was responsible for the production of geraniol in modern roses (Magnard et al., 2015). The NUDX1-1 gene was highly expressed in $\mathrm{OB}$ and in 10 hybrids of the progeny (OW9007, OW9011, OW9013, OW9021, OW9024, OW9047, OW9074, OW9099, OW9149, and OW9204), while NUDX1-2 was expressed in Rw and in 10 hybrids of the progeny (OW9007, OW9013, OW9018, OW9021, OW9024, OW9035, OW9037, OW9082, OW9099, and OW9149) (Figure 2a). Sequencing of the PCR products showed that only RcNUDX1-1a and RwNUDX1-2c were expressed. No correlation was found between the expression of NUDX1-1 and NUDX1-2, suggesting that they have independent functions. Indeed, NUDX1-1 and NUDX1-2 were expressed independently in different individuals, or together as, for example, in OW9099 and others. In addition, the expression levels of NUDX1-2 were generally lower than the expression levels of NUDX1-1 (Wilcoxon rank-sum test, $P<0.01$ ). Differential expression of NUDX1-2 across the hybrids, with a known volatile-associated function of RhNUDX1 (Magnard et al., 2015), could indicate that NUDX1-2 was also associated with the production of one or more volatiles in rose. 
Table 1 Major volatile compounds extracted from petals of $R$. chinensis cv. 'Old Blush' (OB) and R. $\mathrm{x}$ wichurana (Rw) at fully open flower stage (stage four) and analyzed by GC-MS

\begin{tabular}{lll}
\hline & \multicolumn{2}{l}{ Cultivar } \\
\cline { 2 - 3 } Compounds & OB & Rw \\
\hline Z-3-Hexenyl acetate & $5.55^{\mathrm{a}}$ & $\mathrm{nd}$ \\
E-2-Hexenal & 1.21 & 0.48 \\
Z-3-Hexenol & nd & 0.77 \\
Geraniol & 10.96 & $\mathrm{nd}$ \\
Geranial & 4.43 & $\mathrm{nd}$ \\
Germacrene D & 8.42 & $\mathrm{nd}$ \\
$\delta$-Cadinene & 1.24 & $\mathrm{nd}$ \\
E- $\beta$-Farnesene & nd & 0.75 \\
E,E-Farnesol & nd & 2.17 \\
Farnesyl acetate & nd & 0.83 \\
2 PE & nd & 73.04 \\
2PEA & nd & 0.53 \\
TMB & 27.71 & nd \\
Dihydro- $\beta$-ionol & 9.90 & nd \\
\hline
\end{tabular}

TMB， 1,3,5-trimethoxybenzene; 2PE，2-phenylethanol; 2PEA，2phenylethyl acetate; nd, not detected.

${ }^{a}$ Values represent the relative proportion of the total amount (average value of 7-9 different replicates from both 2014 and 2015).

To study this hypothesis, the correlation between the expression levels of the NUDX 1 genes and the volatile profiles of these 16 selected rose hybrids and their parents was analyzed. Spearman's rank correlation test was performed on the expression data of NUDX1-1 and NUDX1-2 with the volatile compounds data from the 18 samples. GC-MS analysis results and correlations are shown in Data $\mathrm{S} 1$. The volatile compounds that showed significant correlation with either NUDX1-1 or NUDX1-2 expression levels are presented in Figure 2b. NUDX1-1 expression had positive correlation with the following monoterpenes: neral $(P \leq 0.001)$, geranial $(P \leq 0.001)$, $\beta$-myrcene $(P \leq 0.001)$, geraniol $(P \leq 0.001)$, $Z$ - $\beta$-ocimene $(P \leq 0.001), E$ - $\beta$-ocimene $(P \leq 0.01)$, and limonene $(P \leq 0.05)$. NUDX1-2 expression showed positive correlation with the following sesquiterpenes: $E, E$-farnesol $(P \leq 0.0001), E, E$-farnesal $(P \leq 0.001)$, $E$ - $\beta$-farnesene $\quad(P \leq 0.001), \quad E, E$ - $\alpha$-farnesene $\quad(P \leq 0.001)$, $Z, E$ - $\alpha$-farnesene $(P \leq 0.001), E$-nerolidol $(P \leq 0.01)$, allofarnesene $(P \leq 0.01)$, $\alpha$-bisabolene $(P \leq 0.05)$, and farnesyl acetate $(P \leq 0.05)$. The strongest correlation was observed with $E$,E-farnesol (Data $\mathrm{S} 1$ ). This result showed that NUDX1-2 is potentially involved in the production of sesquiterpenoids in rose, in particular $E, E$-farnesol.

\section{Quantitative trait locus (QTL) mapping for $E, E$-farnesol content and mapping of the NUDX1-2 gene}

We detected a major QTL for geraniol biosynthesis in the OW progeny on the female linkage group 2, which colocalized with RhNUDX1 (Magnard et al., 2015). In order to further support the hypothesis that RwNUDX1-2 was (a)

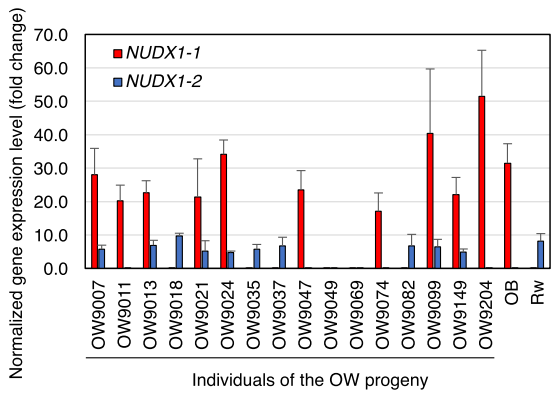

(b)

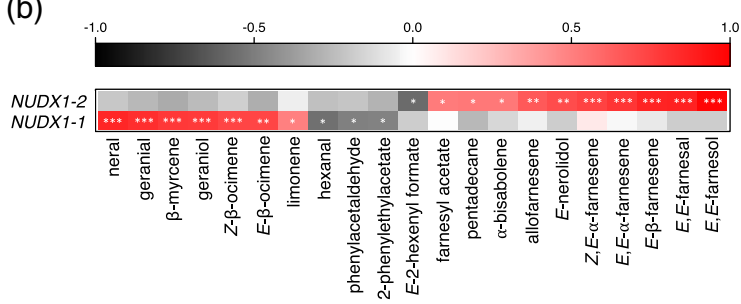

Figure 2. (a) Gene expression levels of NUDX1-1 and NUDX1-2 in $16 \mathrm{~F}$ individuals from a cross between $R$. chinensis cv. 'Old Blush' (OB) and $R$. $x$ wichurana $(\mathrm{Rw})$ and their parents. Transcript levels of NUDX1-1 and NUDX1-2 were normalized to three housekeeping genes coding for $\alpha$-tubulin, elongation factor $1-\alpha$, and translationally controlled tumor protein according to (Dubois et al., 2012). Error bars indicate standard deviation (SD) obtained from two independent biological replicates with at least two technical replicates each. A significant difference was found between the medians of the expression levels of NUDX1-1a and NUDX1-2 (Wilcoxon rank-sum test, $P<0.01$ ). (b) Heatmap correlation (Spearman's rank correlation from -1 to +1 ) of NUDX1-1 and NUDX1-2 expressions with some of the volatile scent compounds that showed significant correlation. ${ }^{*} P \leq 0.05$ ${ }^{*} P \leq 0.01 ;{ }^{* *} P \leq 0.001$. The complete correlation heatmap is available in Data S1.

involved in the production of $E, E$-farnesol and other sesquiterpenoids, we analyzed the distribution of the volatiles produced in petals of all the progeny of the cross between $\mathrm{OB}$ and Rw. Petals from 148 and 132 individuals of the progeny were collected in 2014 and 2015, respectively. Petals were subjected to hexane extraction and extracts were analyzed by GC-MS (Data S2). In total, 104 compounds were identified among 153 individuals (including the parents of the population, $O B$ and $R w$ ), but only 100 compounds were recovered in the samples collected in 2015. The compounds with the highest correlation coefficient with NUDX1-2 expression (Figure 2b, E,E-farnesol, $E, E$-farnesal, $E$ - $\beta$-farnesene, $E, E$ - $\alpha$-farnesene, and $Z, E$ - $\alpha$-farnesene) were chosen for further analyses. $E, E$-farnesol and other sesquiterpenoid contents appeared to segregate in the progeny (Figure S2). Due to the non-normal distribution of the variance residue of data, a log transformation was applied to make the variance residue distribution normal. The raw data were analyzed first by the nonparametric Kruskal-Wallis rank-sum test (KW) (Table S4). Interval mapping analysis was then performed on the 
log-transformed data, for the chromosome regions on which QTLs were detected by KW both in 2014 and 2015 (Table S5). A step size of $1 \mathrm{cM}$ was chosen to find regions with potential QTL effects, i.e., where the LOD score was greater than the threshold. QTLs were detected on linkage group B7 for all the five compound contents (Table S5 and Figure S3). For example, in 2014, E,E-farnesol biosynthesis was detected on the male linkage group B7 (LG B7) at position $51.173 \mathrm{cM}$ (with the marker Rh12GR_21458_519) with a LOD score higher than 20 , explaining $49.9 \%$ of the observed variation in $E, E$-farnesol content (Figure 3b). Next, we developed a genetic marker for RwNUDX1-2, and mapped the gene on the LG B7 at the same position of the Rh12GR_21458_519_marker (Figure 3a). In the reference sequence (homozygous genome from OB, Hibrand SaintOyant et al., 2018; Raymond et al., 2018), no NUDX1-2 sequence was detected in this region (Figure S4). In the heterozygous sequence of OB (Raymond et al., 2018), RcNUDX1-2c (RcHt_S2031.3) is located on the scaffold 2031. The scaffold 2031 is syntenic with a region of chromosome 7 (between 36.2 and $37.0 \mathrm{Mb}$, Figure S4) at a position close to the peak of the QTL (marker Rh12GR_21458_159 located at position $4.38 \mathrm{Mb}$ of the chromosome 7). These results clearly demonstrate that NUDX1-2c co-localized with the QTL for E-E-farnesol production.

(a)

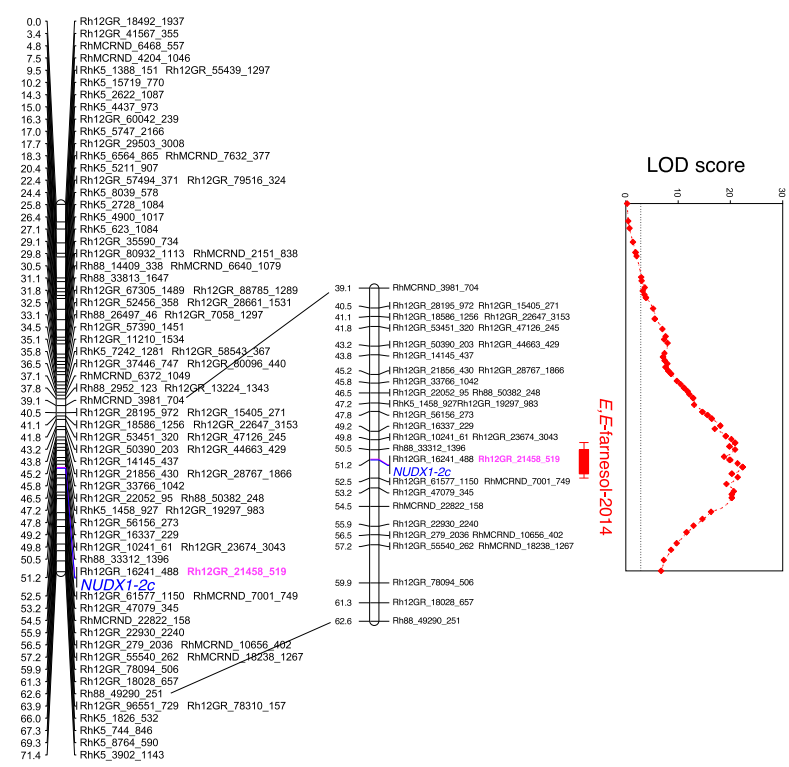

Figure 3. (a) Genetic map of the male linkage group 7, based on SNP and RwNUDX1-2 markers. The genetic region where the QTL for $E, E$-farnesol production is located is enlarged to see the position of the markers. (b) $A$ LOD score curve obtained from interval mapping analysis of $E, E$-farnesol contents in flowers on the male linkage group B7. The dashed line indicates the genome-wide significant threshold based on a permutation test. The marker framed in pink (Rh12GR_21458_519) indicates the highest LOD obtained in this analysis. The unit for the locus axis is centimorgan (cM). Volatile contents were analyzed in 2014

\section{Functional characterization of recombinant NUDX1 proteins in vitro and in vivo}

To test the function of NUDX1-2, in vitro protein assays and in vivo transient transformation assays were performed. For the protein assay, five NUDX1 proteins were expressed and purified using a bacterial Rosetta ${ }^{\circledR}$ system, including two NUDX1-1 sequences from OB (RcNUDX1-1a and RcNUDX1-1b), one from Rw (RwNUDX1-2c), one from PM (RhNUDX1), and one from $A$. thaliana (AtNUDX1). Primers for gene cloning into expression vectors are listed in Table S2. Each purified protein was incubated with five potential substrates: GPP, FPP, IPP, dGTP, and 8-oxo-dGTP; the results are presented in Table 2.

All NUDX1 proteins could convert GPP, FPP, and IPP into related products, but only AtNUDX1 could use dGTP and 8-oxo-dGTP as substrates (Table 2). Among all NUDX1-1 proteins, RcNUDX1-1b and AtNUDX1 exhibited higher $k_{\text {cat }} / K_{M}$ values on both GPP and FPP than those of the other rose proteins, indicating that AtNUDX1 and RcNUDX1-1b could use these substrates with higher efficiency in vitro. Most NUDX1-1 proteins had similar $k_{\text {cat }}$ $K_{M}$ values for GPP and FPP, indicating that they had similar reaction efficiencies on these two substrates in vitro. For RwNUDX1-2c, however, the $k_{c a t} / K_{M}$ value for FPP was 140 times higher than that for GPP, indicating that NUDX1-2 preferred FPP over GPP in vitro. In addition, all proteins accepted IPP as substrate. Unlike AtNUDX1, rose NUDX1 proteins did not accept dGTP and 8-oxo-dGTP as substrates. However, due to the liquid chromatography LC-MS method used for activity measurements, kinetics parameters could not be determined for IPP and 8-0xodGTP substrates.

In order to obtain more evidence for the function of RwNUDX1-2, transient transformation of RwNUDX1-2c (35S:RwNUDX1-2c) in Nicotiana benthamiana leaves was conducted, in parallel with transient transformation of RhNUDX1 (35S:RhNUDX1, positive control) and GFP (35S: GFP, negative control). Three days after transformation, infiltrated leaves were collected and freeze-dried, followed by extraction and analysis of geraniol and farnesol glycosides using ultrahigh-performance LC-MS. Very small quantities of geraniol and farnesol glycosides were found in the leaf samples that were infiltrated with 35S:GFP construct. A significant amount of geraniol glycosides was detected in the leaf samples that were infiltrated with 35S: $R h N U D X 1$ construct (Student $t$ test, $P \leq 0.001$ ) (Figure 4). The amounts of farnesol glycosides were of the same order as that of the control leaves. Interestingly, significant amounts of both geraniol and farnesol glycosides were found in the leaf samples that were infiltrated with 35S: $R w N U D X 1-2 c$ (Student $t$ test, $P \leq 0.001$ ), indicating that the RwNUDX1-2 protein is involved in the production of farnesol and geraniol in vivo. 
Table 2 Kinetic parameters of NUDX1 proteins: RcNUDX1-1a, RcNUDX1-1b, RhNUDX1, RwNUDX1-2c, and AtNUDX1, with several potential substrates

\begin{tabular}{|c|c|c|c|c|c|}
\hline Protein & Substrates & Activities $^{a}$ & $K_{M}(\mathrm{M})$ & $k_{\text {cat }}\left(\sec ^{-1}\right)$ & $k_{\text {cat }} / K_{M}\left(\mathrm{M}^{-1} \sec ^{-1}\right)$ \\
\hline \multirow[t]{5}{*}{ RcNUDX1-1a } & GPP & + & $1.96 \times 10^{-6}\left(1.36 \times 10^{-7}\right)$ & $0.20(0.09)$ & $1.02 \times 10^{5}$ \\
\hline & FPP & + & $4.00 \times 10^{-6}\left(1.01 \times 10^{-6}\right)$ & $0.73(0.48)$ & $1.82 \times 10^{5}$ \\
\hline & IPP & + & n.c. ${ }^{b}$ & n.c. & n.c. \\
\hline & dGTP & - & n.d. ${ }^{c}$ & n.d. & n.d. \\
\hline & 8-oxo-dGTP & - & n.d & n.d. & n.d. \\
\hline \multirow[t]{5}{*}{ RcNUDX1-1b } & GPP & + & $2.61 \times 10^{-7}\left(1.10 \times 10^{-7}\right)$ & $0.30(0.09)$ & $1.15 \times 10^{6}$ \\
\hline & FPP & + & $5.55 \times 10^{-7}\left(6.86 \times 10^{-8}\right)$ & $1.37(0.81)$ & $2.47 \times 10^{6}$ \\
\hline & IPP & + & n.c. & n.c. & n.c. \\
\hline & dGTP & - & n.d. & n.d. & n.d. \\
\hline & 8-oxo-dGTP & _- & n.d. & n.d. & n.d. \\
\hline \multirow[t]{5}{*}{ RhNUDX1 } & GPP & + & $1.13 \times 10^{-6}\left(4.13 \times 10^{-7}\right)$ & $0.29(0.11)$ & $2.57 \times 10^{5}$ \\
\hline & FPP & + & $1.54 \times 10^{-6}\left(1.88 \times 10^{-7}\right)$ & $1.21(0.74)$ & $7.86 \times 10^{5}$ \\
\hline & IPP & + & n.c. & n.c. & n.c. \\
\hline & dGTP & - & n.d. & n.d. & n.d. \\
\hline & 8-oxo-dGTP & - & n.d. & n.d. & n.d. \\
\hline \multirow{5}{*}{ RwNUDX1-2 } & GPP & + & $8.78 \times 10^{-6}\left(9.01 \times 10^{-7}\right)$ & $0.21(0.09)$ & $2.39 \times 10^{4}$ \\
\hline & FPP & + & $4.95 \times 10^{-7}\left(7.90 \times 10^{-8}\right)$ & $1.67(1.51)$ & $3.37 \times 10^{6}$ \\
\hline & IPP & + & n.c. & n.c. & n.c. \\
\hline & dGTP & - & n.d. & n.d. & n.d. \\
\hline & 8-oxo-dGTP & - & n.d. & n.d. & n.d. \\
\hline \multirow[t]{5}{*}{ AtNUDX1 } & GPP & + & $1.38 \times 10^{-7}\left(4.86 \times 10^{-8}\right)$ & $0.26(0.10)$ & $1.88 \times 10^{6}$ \\
\hline & FPP & + & $4.84 \times 10^{-7}\left(1.05 \times 10^{-7}\right)$ & $1.91(0.45)$ & $3.95 \times 10^{6}$ \\
\hline & IPP & + & n.c. & n.c. & n.c. \\
\hline & dGTP & + & n.c. & n.c. & n.c. \\
\hline & 8-oxo-dGTP & + & n.c. & n.c. & n.c. \\
\hline
\end{tabular}

Data are presented as the means of three to six replicates using native protein, and the standard deviation (SD) is indicated between brackets. FPP, farnesyl diphosphate; GPP, geranyl diphosphate; dGTP, deoxyguanosine triphosphate; IPP, isopentenyl diphosphate; 8-oxo-dGTP, 7,8-dihydro-8-oxo-deoxyguanosine triphosphate.

${ }^{a}$ Activities indicate the interaction between the protein and the substrate, + means interaction was detected and - means no interaction was detected.

${ }^{b}$ n.c., not calculated, which means that there was a detectable activity but data could not be used for calculation.

${ }^{c}$ n.d., not detected, which means that activity was too low to be detected with given substrates.

\section{Determination of the crystal structure of the rose NUDX1- 1 protein}

As demonstrated above, RhNUDX1, RcNUDX1-1a, RcNUDX1-1b, RwNUDX1-2c, and AtNUDX1 can interact with FPP, GPP, and IPP (Table 2). However, rose NUDX1 seem to have lost their function on oxidized nucleotides (e.g., 8-oxo-dGTP). Moreover, NUDX1-2 seems to be more active on FPP than on GPP, which is not the case for the other rose proteins. In order to study the NUDX1-substrate interactions and to find out if the 3D structure was responsible for the different substrate specificities, the crystallization of rose NUDX1 protein was carried out. NUDX1 protein requires cations for the catalytic reaction. Divalent cations were therefore excluded during crystallization assays to inactivate the enzyme and to capture the substrate bound to the enzyme. The structure of RhNUDX1, the first structure of a Nudix enzyme from rose, was thus solved in the absence and in the presence of the GPP substrate (Figure 5a). The RhNUDX1 structure without any substrate was refined at $1.7 \AA$ resolution (Table S6) with one molecule per asymmetric unit in the protein crystal. In contrast to AtNUDX1, RhNUDX1 seems to be monomeric in solution, as the analysis using PISA (Krissinel and Henrick, 2007) of intermolecular contacts in the crystal did not identify assemblies of higher order. RhNUDX1 harbors the Nudix fold $(\alpha / \beta / \alpha$ sandwich structure). The GPP-bound structure of RhNUDX1 was obtained by flash-soaking crystals of RhNUDX1 in a solution of GPP and was refined at $1.45 \AA$ resolution (Table S6). No structural difference was detected when comparing the structures of RhNUDX1 with and without GPP (root mean square deviation [RMSD] of $\mathrm{C} \alpha, 0.19 \AA$ ) (Figure 5a). The X-loop (amino acids from position 86 to 90 ), which connects $\beta 5$ and $\beta 6$ strands and which is less conserved in the NUDX1 family, has been previously proposed to carry the substrate specificity (Srouji et al., 2017). This loop seems to be more dynamic in the presence of GPP and does not participate in the binding of GPP (Figure 5a). The GPP substrate showed a well-defined electron density map in the active site of RhNUDX1 (Figure 5b). 


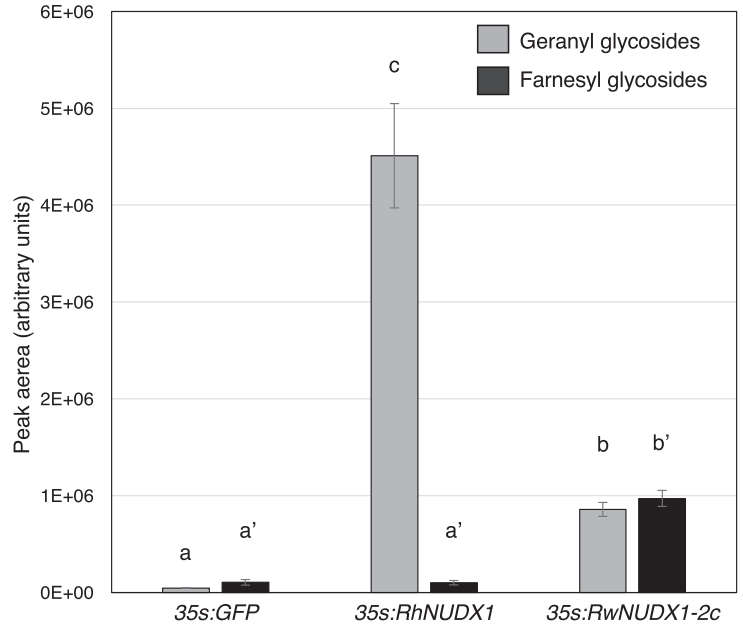

Figure 4. Patterns of terpenol glycoside accumulation following transient expression of NUDX1 genes in $N$. benthamiana. Leaves of $N$. benthamiana were transformed with a NUDX1 construct (35S:RwNUDX1-2c or 35: RhNUDX1) or with the 35S:GFP control. For each construct, eight independent biological replicates were used to quantify relative amounts of terpenol glycosides. Relative amounts are given as mean peak areas corresponding to the $[\mathrm{C} 10 \mathrm{H} 17]^{+}$ion $(\mathrm{m} / \mathrm{z}$ 137.1325) for geranyl glycosides and to the $[\mathrm{C} 15 \mathrm{H} 24]^{+}$ion $(\mathrm{m} / \mathrm{z} 205.1952)$ for farnesyl glycosides (expressed as arbitrary units); bars indicate the standard error. Means with different letters are significantly different (Student's $t$ test, $P<0.01$ ).

The structure of the RhNUDX1/GPP complex is remarkably similar to the structure of the AtNUDX1/GPP complex (PDB code 5GP0, Liu et al., 2018), with an RMSD of $\mathrm{C} \alpha$ atoms of $0.75 \AA$ (Figure $5 \mathrm{c}$ ). In these structures, GPP is nearly at the same position. Both structures were solved in the absence of cations (due to the absence of cations in the crystallization assays for RhNUDX1 and an E56A mutation in the Nudix motif of AtNUDX1 to prevent cation binding). In the RhNUDX1/GPP complex, the two phosphate groups of GPP make polar contacts with residues H49, Y94, S47, and R34 and the aliphatic chain of GPP sits in a hydrophobic pocket (Figure 6a). Although GPP is bound in the active site, it is not in a position to be hydrolyzed, as suggested by the structure of complexes of AtNUDX1/IPP (Henry et al., 2018) and AtNUDX1/8-oxo-dGTP solved with $\mathrm{Mg}^{2+}$ bound in the active of the enzymes (Jemth et al., 2019). Therefore, we turned to molecular modeling to further investigate and understand the substrate discrimination by rose NUDX1 proteins.

\section{Modeling of rose NUDX1 proteins}

From the newly determined RhNUDX1 crystal structure, we conducted molecular modeling on rose NUDX1 proteins, based on the available crystal structures of AtNUDX1 complexed with 8-oxo-dGTP (Jemth et al., 2019), with IPP (Henry et al., 2018), and with GPP (Liu et al., 2018), of E. coli MutT complexed with 8-oxo-dGMP (Nakamura et al., 2010), and of human MTH1 complexed with 8-oxo- (a)

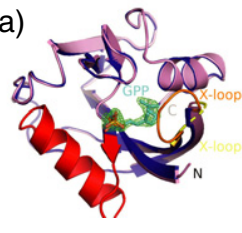

(b)

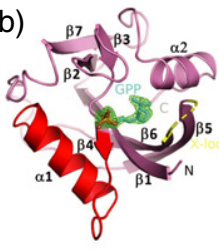

(c)

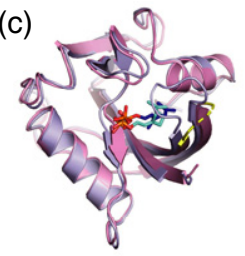

Figure 5. (a) Superimposition of the RhNUDX1/GPP complex (pink) and apo RhNUDX1 (blue). The X-loop for the apo structure is colored in orange. (b) Structure of the RhNUDX1/GPP complex. The electron density map contoured at $1.0 \sigma$ is shown around the GPP ligand colored in cyan. The secondary structure elements are numbered according to Figure S5, and the NUDX box (part of $\beta 4$-loop- $\alpha$ 1-loop) and the X-loop are colored in red and yellow, respectively. (c) Superimposition of RhNUDX1/GPP (pink) and AtNUDX1/GPP (PDB code 5GP0) (light blue). GPP is shown as sticks in cyan (RhNUDX1) and blue (AtNUDX1).

GMP (Svensson et al., 2011). Models of others Rose NUDX1 were obtained by structural homology from crystal structures of NUDX1 proteins. The amino acid sequences of rose NUDX1 proteins were first aligned with AtNUDX1, MutT, and MTH1 according to sequence conservation and secondary structure and manually adjusted in order to have a correct structure-based sequence alignment (Figure S5). The AtNUDX1/IPP (PDB code 6DBZ) and AtNUDX1/ 8-oxo-dGTP (PDB code 6FL4) structures contain two $\mathrm{Mg}^{2+}$ ions in the active site, stabilizing the interaction with their substrates. These structures were used to position $\mathrm{Mg}^{2+}$ ions and ligands in the active sites of NUDX1 proteins. Molecular dynamics (MD) trajectories were calculated for $20 \mathrm{~ns}$ and were analyzed for $\mathrm{C} \alpha \mathrm{RMSD}$, protein residue fluctuation (RMSD), ligand position RMSD, the distance between $\mathrm{Mg}^{2+}$ and the phosphate group, and predicted binding energy (Figure S6 and Table S7). The values calculated for the analysis of MD trajectories showed limited variations over time, suggesting that the structures modeled after energy minimization and 20 ns MD represent stable ligand-bound states. The representative structures, corresponding to the mean binding energy at the end of the MD trajectories, were compared.
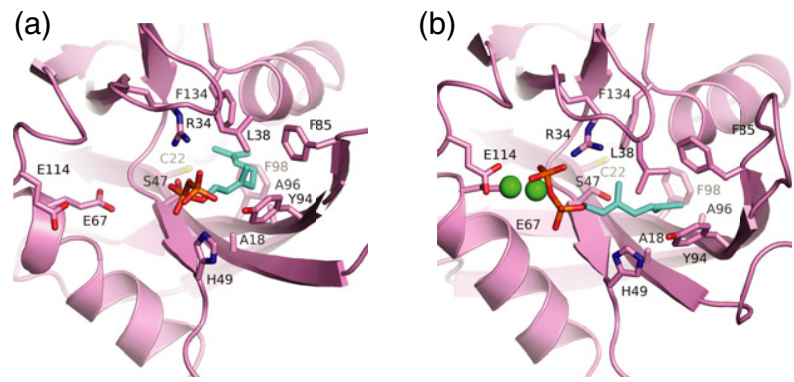

Figure 6. Comparison of GPP ligand position (a) in the crystal structure of RhNUDX1 and (b) in the model obtained by molecular modeling. The geranyl moiety is shown in cyan and the phosphate moiety in orange. $\mathrm{Mg}^{2+}$ ions are shown in green. The side chains of the residues of the ligand binding site are shown in sticks. 
The analysis of averaged values produced during MD simulations (Table S7) show that AtNUDX1 exhibits the lowest predicted binding energy for 8-oxo-dGTP, whereas RwNUDX1-2c shows the lowest predicted binding energy for FPP among the rose NUDX. This indicates that AtNUDX1 presents a higher affinity for 8-oxo-dGTP and RwNUDX1-2C for FPP, which is in good agreement with the data in Table 2. MD models show a good fit with known crystal structures. Indeed, in models of AtNUDX1/8oxo-dGTP and AtNUDX1/GPP, ligands are very close to their position in the crystal structures (PDB codes 6FL4 and $6 \mathrm{DBZ}$, respectively) (Figure S6a,d). For the RhNUDX1/GPP model, although the ligand is nearly at the same position (Figure $6 \mathrm{~b}$ and Figure S7e), GPP is moved closer to the $\mathrm{Mg}^{2+}$ coordination sites, confirming that in the crystal structures of RhNUDX1/GPP and AtNUDX1/GPP (5GPO), GPP is too far from the $\mathrm{Mg}^{2+}$ coordination sites for hydrolysis. Thus, MD provides a model for the RhNUDX $1 / \mathrm{Mg}^{2+}$ / GPP complex that is relevant for the hydrolysis of the phosphate group and compatible with geranyl chain position (Figure 6b).

The binding site pockets with their equivalent amino acids for AtNUDX1, RhNUDX1, and RwNUDX1-2c are shown in Figure S7. In order to find differences in the active sites of these proteins that could explain their substrate preferences, only amino acids which are not conserved in these proteins are discussed below. Five residues that are not conserved and that can account for substrate preferences were identified in the substrate pocket of these proteins, namely, A11, F15, G40, S89, and S91 in AtNUDX1; A18, C22, S47, A96, and F98 in RhNUDX1; and V19, C23, G48, V97, and S99 in RwNUDX12c. In addition, it has been shown in AtNUDX1 that N76 and $\mathrm{S} 89$, which are in the binding site pocket, play a role in 8-oxo-dGTP hydrolysis (Jemth et al., 2019). The position corresponding to N76 is conserved in all NUDX studied (Figure S5, N83 in RhNUDX1). However, there is no serine at the position corresponding to $\mathbf{S} 89$ for rose enzymes. This position is occupied either by an alanine residue (A96 in RhNUDX1, RcNUDX1-1a, and RcNUDX1-1b) or a valine residue (V97 in RwNUDX1-2c). This amino acid substitution may account for a lower activity towards 8-oxo-dGTP and could explain differences in substrate preferences. Indeed, S89 establishes a hydrogen bond with oxygen from 8-oxo-dGTP (Figure S7), which cannot happen with V97 or A96.

Moreover, the binding pocket is slightly different in RwNUDX1-2c compared to other NUDX1. RwNUDX1-2c has a valine residue (V97) which is bulkier than the corresponding alanine present in other rose NUDX. We already mentioned that this position was shown to be important for interaction with 8-oxo-dGTP in AtNUDX1 (Jemth et al., 2019). In RwNUDX1-2c, V97 may render the binding pocket more hydrophobic. RwNUDX1-2c also has a larger ligand binding site and could fit the longer FPP aliphatic chain. The ligand binding pocket is narrower in AtNUDX1 and in RhNUDX1 due to the presence of F15 and F98, respectively, while these positions are occupied by $\mathrm{C} 23$ and S99 in RwNUDX1-2c. Therefore, a subtle combination of amino acids in the substrate binding pocket may account for the observed substrate specificities.

\section{DISCUSSION}

Not so long ago, NUDX1 proteins were generally considered as 'house cleaning' enzymes (Bessman et al., 1996; Yoshimura et al., 2007; Bessman, 2019). Indeed, NUDX1 proteins from $E$. coli and humans have been shown to play major roles in removing oxidized nucleotides (Setoyama et al., 2011; Gad et al., 2014). The human protein MTH1 is critical for cancer cell survival where oxidative damage is very high, and targeting this protein is a highly promising anticancer strategy (Carter et al., 2015). However, involvement of a NUDX1 protein in scent production has only been described in roses recently (Magnard et al., 2015). Since this initial discovery, several studies have shown that in plants, these proteins may have evolved to fulfill other roles, apart from sanitizing the cell from oxidized nucleotides. In Arabidopsis, two Nudix hydrolases, among which AtNUDX1, were shown to play a role in terpene biosynthesis, by regulating the ratio of their precursors, IPP and dimethylallyl diphosphate (Henry et al., 2018). It is not known if the two functions, sanitization of nucleotide pools and regulation of terpene biosynthesis, are both performed by AtNUDX1 in vivo, perhaps in different tissues. It was shown that AtNUDX1 activity on 8-oxo-dGTP is poor compared to that of human MTH1 (Jemth et al., 2019). Nevertheless, AtNUDX1 is the only one of 25 Nudix hydrolases present in Arabidopsis able to act on mutagenic derivatives of dGTP and GTP and it is fully able to complement MutT mutation in E. coli (Ogawa et al., 2005; Ogawa et al., 2008).

In this work, we investigated the molecular bases of the peculiar scent composition of Rw, whose petals emit significant amounts of $E, E$-farnesol and other sesquiterpenes. To this end, we characterized $F_{1}$ individuals in a cross between $\mathrm{OB}$ and $\mathrm{Rw}$, two rose cultivars with striking differences in scent composition. We found that only two genes were highly expressed in the petals of the parental lines $\mathrm{OB}$ and Rw, namely, RcNUDX1-1a and RWNUDX1-2c, respectively. Expression of the RcNUDX1-1a gene correlated with the production of geraniol in the OW progeny. This finding was expected because RcNUDX1-1a was very similar to RhNUDX1 from R. x hybrida cv. 'Papa Meilland', which has been shown to be responsible for the biosynthesis of monoterpenes, especially geraniol in this highly fragrant cultivar (Magnard et al., 2015). Interestingly, expression of RwNUDX1-2c correlated with the production of $E, E$-farnesol and other sesquiterpenoids in the OW progeny. A major OTL governing the production of $E, E-$ 
farnesol was detected, which co-localized with RhNUDX1$2 c$, providing strong evidence that polymorphism at the NUDX1-2c locus was responsible for the difference of $E, E$ farnesol production in the OW progeny. We also have shown that there is a link between NUDX1-2 expression and the presence of linear sesquiterpenes such as $E, E$-afarnesene and $E$ - $\beta$-farnesene. As QTLs for these compounds co-localized with the QTL for farnesol, we can assume that this locus is involved in the biosynthesis of these compounds as well. We do not have mechanistic data to explain this finding. FP could be converted to these products by an uncharacterized pathway. Alternatively, these compounds could be degradation products arising from farnesol in planta or during the extraction process. A terpene synthase has been previously shown to be responsible for the production of the sesquiterpene germacrene $D$ in roses (Guterman et al., 2002). It is interesting to see that expression of NUDX1-2 in the progeny did not correlate with all sesquiterpenes; for example, it was not correlated with the production of germacrene D (Data S1). Therefore, it seems that in rose petals, two different pathways may operate for the production of sesquiterpenoids.

In vitro assays showed that the recombinant rose NUDX1 proteins could hydrolyze GPP, FPP, and IPP into GP, FP, and IP respectively. GP has been shown to be further hydrolyzed by an unidentified phosphatase (Magnard et al., 2015), which may also be the case for FP and IP. The RcNUDX1-1a and RcNUDX1-1b proteins did not show a marked preference for GPP or FPP in vitro. Conversely, RwNUDX1-2 used FPP more efficiently than GPP. Expression of RhNUDX1 led to the accumulation of geranyl glycosides in tobacco leaves, without detectable biosynthesis of farnesyl glycosides, suggesting a strict selection of the GPP substrate in vivo. Conversely, expression of RwNUDX1-2 led to accumulation of both farnesyl and geranyl glycosides, indicating a relaxed substrate preference in vivo, which may reflect differences in GPP and FPP substrates availability in $N$. benthamiana leaves. Indeed, GPP and FPP availabilities in the $R w$ rose cultivar could be major factors impacting RwNUDX1-2c substrate preference in planta. Nevertheless, experiments in $N$. benthamiana showed that the biosynthesis of significant amounts of $E$, $E$-farnesol derivatives in vivo was specific to the RwNUDX1-2 protein. Taken together, the activity of the RwNUDX1-2 in vitro and in planta and the co-localization of the corresponding gene with a QTL for E,E-farnesol accumulation are consistent with RwNUDX1-2 being involved in the biosynthesis of $E, E$-farnesol and other sesquiterpenoids in the petals of Rw.

$E, E$-farnesol is a sesquiterpene alcohol with a mild, delicate, sweet-oily odor (Lapczynski et al., 2008). It is used as an ingredient for perfume and cosmetic products, such as deodorants and bath products. It may be present in high amounts in petals of some flowers such as Actinidia chinensis (Green et al., 2012). In plants, only five farnesol synthases have been characterized so far (Schnee et al., 2002; Cheng et al., 2007; Parveen et al., 2015; Chen et al., 2016; Rusdi et al., 2018), and three of them produce a blend of sesquiterpenes when provided with the substrate FPP. In the present work, we show that, besides terpene synthases, Nudix hydrolases may be involved in the biosynthesis of sesquiterpenes, as shown previously in the case of monoterpenes (Magnard et al., 2015).

Though several crystal structures of plant NUDX have been solved with various substrates, the structural basis for substrate preference is still under investigation. In this paper, MD helped to understand the catalytic mechanism of NUDX1. Key amino acids for substrate binding were identified. Based on the results from MD, two positions corresponding to S89 and S91 in AtNUDX1, A96 and F98-in RhNUDX1, and V97 and S99-in RwNUDX1-2c could be essential to explain RwNUDX1-2c preference for FPP. However, although the important S89 in AtNUDX1, which is $\mathrm{H}$ bonded to 8-oxo-dGTP, is absent in the rose NUDX, the structural modeling could not fully explain why AtNUDX1 is the only enzyme which can hydrolyze 8-oxo-dGTP. Sequence comparison of RcNUDX1-1a, RcNUDX1-1b, RhNUDX1, RwNUDX1-2c, and AtNUDX1 also revealed differences in the X-loop region (86-91 with sequence LDEAKP in RhNUDX1) (Figure S5). This loop, which is well defined in the RhNUDX1 apo structure but which is disordered in RhNUDX1-GPP crystal structures, is one of the most flexible parts during MD simulations, as plotted on the protein $\mathrm{C} \alpha$ fluctuation diagram (Figure S6). It may also interact with the substrate, but the precise role of the $\mathrm{X}$-loop is not known. It has been suggested that modifications within this region could alter substrate specificity, thus allowing for altered substrate specificity and neofunctionalization (Srouji et al., 2017). NUDX1 proteins can have very different binding mechanisms even if they use the same substrates. It has been shown that the substrate-interacting amino acids are completely different when comparing MTH1 and MutT (Svensson et al., 2011), which makes the identification of key amino acids quite complicated. Although there are no specific residues in the X-loop which interact with the ligand, the flexibility and dynamics of this loop may still be important for ligand turnover.

We showed here that in Rw, the NUDX1-2 protein plays a role in the biosynthesis of sesquiterpenoid scent compounds, as a result of functional diversification in the Nudix hydrolase gene family. It remains to be established whether the NUDX1-dependent scent biosynthetic pathways are specific to roses, as they have not been characterized in other plant species so far. In many plants, such as rice (Oryza sativa) and maize (Zea mays), biosynthesis of farnesol is catalyzed by typical terpene synthases (Schnee et al., 2002; Cheng et al., 2007). It is interesting to note that farnesol is a precursor of the juvenile hormone in insects 
(Bellés et al., 2005; De Loof and Schoofs, 2019). In some of these insects, it has been shown that farnesol is derived from FPP with the assistance of phosphatases from the haloalkanoic acid dehalogenase superfamily (Cao et al., 2009; Nyati et al., 2013). In recent years, the importance of the convergence of multiple unrelated metabolic pathways towards the biosynthesis of a single compound has been increasingly acknowledged (Sun et al., 2016). The biosynthesis of farnesol is therefore another striking example of such metabolic convergence.

\section{EXPERIMENTAL PROCEDURES}

\section{Plant material}

A population of $151 \mathrm{~F}_{1}$ progeny was generated by crossing two diploid roses (Hibrand Saint-Oyant et al., 2018): R. chinensis cv. 'Old Blush' (OB, maternal plant) and $R . \mathrm{x}$ wichurana (Rw, paternal plant) originated from the Bagatelle garden (Paris, France). All these rose plants were grown outside at INRA Angers (Experimental Unit Hortis, Angers, France). Petals of all descendants were collected between May and July in 2014 and 2015. Progeny (148 and 132 individuals) were collected in 2014 and 2015, respectively. This difference in sample size is because some roses that were available in 2014 were dead in 2015 or too young to have flowers in 2014 and became capable of blooming in 2015. Collected petals were subjected to hexane extraction and extracts were analyzed by GC-MS. Transient transformation assays were performed on the leaves of 4-week-old $N$. benthamiana plants that were grown inside a climate room $\left(21 \pm 1{ }^{\circ} \mathrm{C}, 16\right.$-h light period and 8-h dark period).

\section{Total RNA extraction and primary cDNA synthesis}

Sixteen out of the 156 progeny and the two parents were selected for total RNA extraction: OW9007, OW9011, OW9013, OW9018, OW9021, OW9024, OW9035, OW9037, OW9047, OW9049, OW9069, OW9074, OW9082, OW9099, OW9149, OW9204, OB, and Rw. The rose petals of these selected plants were collected and frozen immediately in liquid nitrogen. Prior to RNA extraction, frozen rose petals were ground to fine powder in liquid nitrogen using a sterilized mortar and pestle. Approximately $300-400 \mathrm{mg}$ (3-4 tubes containing $100 \mathrm{mg} \pm 15 \%$ ) of frozen ground petals was used in order to obtain sufficient amounts of total RNA. Total RNA was extracted using the NucleoSpin ${ }^{\circledR}$ RNA plant kit (MACHEREYNAGEL, Düren, Germany) according to the manufacturer's instructions with slight modification as follows: before adding the lysis buffer (RAP), a spatula tip (around $5 \%$ of the sample weight) of polyvinylpyrrolidone (PVP-40, Sigma-Aldrich, St. Louis, USA) and ethylhexadecyldimethyl ammonium bromide (CTAB) (SigmaAldrich) was added directly to the ground petals, followed by $5 \mu \mathrm{l}$ of $\beta$-mercaptoethanol ( $\geq 99.0 \%$, Sigma-Aldrich). RNA was dissolved in the mixture at $60^{\circ} \mathrm{C}$ for 5 min and subsequently extracted using 1 volume of chloroform or chloroform:isoamyl alcohol (24:1) (Sigma-Aldrich). Supernatant was transferred to a new tube for further procedures. Prior to the loading, 0.5 volume of $100 \%$ ethanol was added in order to adjust the binding condition. The remaining washing procedures were performed according to the manufacturer's protocol, including decontamination of genomic DNA using DNase.

The quality of the RNA samples was evaluated by absorbance measurements using a NanoDrop 2000c (Thermo Fisher Scientific, Waltham, USA), and the integrity was determined by electrophoretic analysis. All the RNA samples used for the qRTPCR reactions had a $260 / 280 \mathrm{~nm}$ absorbance ratio between 1.7 and 2.2, while RNA samples with a 260/280-ratio close to 2 were generally qualified for subsequent reactions. To rule out any genomic DNA contamination in the RNA extracts, the RNA samples were subjected to PCR amplifications of the Tubulin and NUDX1 gene (35 cycles). No visible amplifications of genomic DNA were detected from the RNA samples. The primary cDNA was synthesized from approximately $2 \mu \mathrm{g}$ of total RNA using the SuperScript ${ }^{\circledR}$ III reverse transcriptase, oligo-dT, and RNasOUT ${ }^{\mathrm{TM}}$ recombinant RNase inhibitor (all mentioned products above are from Thermo Fisher Scientific) in a reaction volume of $20 \mu \mathrm{l}$ according to the manufacturer's procedure. The quality of the synthesized cDNA was tested using PCR with the primers of the housekeeping gene castor bean (Ricinus communis) translationally controlled tumor protein (RCTCTP). If an amplicon was found after 28 cycles of amplification, then the quality of cDNA sample was assumed good enough for further analysis.

\section{qRT-PCR analysis}

The reaction mixture of primary cDNA was diluted 125 times before qRT-PCR, which was carried out with a CFX96 ${ }^{\mathrm{TM}}$ Real-Time system equipped with $\mathrm{C}_{1000^{\mathrm{TM}}}$ Thermal Cycler (Bio-Rad, California, USA). The qRT-PCR reaction mixture consisted of $10 \mu \mathrm{l}$ of SsoAdvanced ${ }^{\text {TM }}$ SYBR ${ }^{\circ}$ Green Supermix (Bio-Rad), $2 \mu \mathrm{l}$ of a pair of primers $(1 \mu \mathrm{l}$ for each primer, final primer concentration: $10 \mu \mathrm{m})$, $2 \mu \mathrm{l}$ of diluted cDNA mixture, and DNase-free water to reach a total volume of $20 \mu \mathrm{l}$. The thermal cycling profile applied was $95^{\circ} \mathrm{C}$ for $5 \mathrm{sec}$, followed by $30 \mathrm{sec}$ at 58,60 , or $64^{\circ} \mathrm{C}$ depending on the primer pair. In total 40 cycles were performed for each batch of samples. Within each qRT-PCR batch, two negative controls with water instead of cDNA were used. All qRT-PCR were carried out in two biological replicates, each of which was subjected to RNA extraction followed by qRT-PCR in two technical replicates. The resulting quantification cycle $(\mathrm{Cq})$ values were the mean of four values from two biological and two technical replicates. These $\mathrm{Cq}$ values were automatically determined by the $\mathrm{CFX} 6^{\mathrm{TM}}$ Real-Time system with default settings. In order to evaluate the changes in expression levels of certain genes, three housekeeping genes that have consistent transcription levels across different samples were used as reference genes, i.e., genes that code for $\alpha$-tubulin, elongation factor 1-alpha, and TCTP according to Dubois et al. (2012). To obtain the relative quantities of each amplified product in the samples (relative to the reference genes), the $\Delta \Delta \mathrm{Ct}$ method was applied (Pfaffl, 2001). The melting curves of the amplified products were analyzed to determine the specificity of each qRT-PCR reaction, using the built-in standard method in the system. The primers that were used for RT-PCR and QRT-PCR are listed in Table S2.

\section{RNA sequencing and assembly}

Extracted total RNAs of OB, Rw, OW9035, and OW9047 hybrids with RNA integrity number (RIN) higher than 7 were used for RNA sequencing (Eurofins Genomics, Ebersberg, Germany) using an Illumina HiSeq 2500 sequencer with single-read module. The quality of the obtained RNA sequencing reads was assessed using FastQC (http://www.bioinformatics.babraham.ac.uk/projects/fa stqc/), and reads were trimmed to remove sequencing adapters using Trimmomatic (Bolger et al., 2014). This was followed by reassessment using FastQC. The trimmed sequence reads of the above four samples were pooled together and used for de novo transcriptome assembly, using the software suite Trinity (Grabherr et al., 2011). Next, we used the generated transcriptome as a 
reference to map the reads from each individual sample and then estimate contig abundances using the RNA-Seq by Expectation Maximization (RSEM) package (Li and Dewey, 2011). This was followed by gene differential expression analysis in pairs using the edgeR package (Robinson et al., 2010). The trimmed mean of Mvalues (TMM) method (Robinson and Oshlack, 2010) was applied for read counts normalization in FPKM values. Finally, the normalized data were centered by median and $\log _{2}$-transformed to obtain relative expression levels.

\section{QTL analyses and development of a genetic marker for NUDX1-2}

QTL analysis was carried out using MapOTL ${ }^{\circledR} 5.0$ (Van Ooijen, 2004). These QTL analyses were conducted using female and male SNP maps that have been developed using JoinMap 4.0 (Van Ooijen, 2006). Due to non-normality for most of the metabolites, the data were analyzed first by KW. Interval mapping analysis was performed with a step size of $1 \mathrm{cM}$ to find regions with potential QTL effects, i.e., where the LOD score was greater than the threshold. A LOD threshold at which a QTL was declared significant was determined according to a genome-wide error rate of 0.05 over 1000 permutations (Churchill and Doerge, 1994). The percentage explained by the QTL $\left(r^{2}\right)$ was also presented.

Based on the RcNUDX1-2c sequence (RcHt_2031.3), PCR primers (RhNUDX1-2_F1 and RhNUDX1-2_R1, Table S2) were developed and length polymorphism was detected: two DNA fragments (between 700 and $800 \mathrm{bp}$ ) for the locus in Rw and one DNA fragment for the same locus in OB (around $700 \mathrm{bp}$ ). PCR reactions were performed in $25 \mu \mathrm{l}$ volume with $10 \mathrm{ng}$ genomic DNA, 1x Q $5^{\circledR}$ buffer, $0.200 \mathrm{~mm}$ dNTPs, $0.5 \mu \mathrm{m}$ of each primer, and $0.02 \mathrm{U}$ of Q $5^{\circledR}$ High-Fidelity DNA Polymerase (New England Biolabs, Ipswich, USA), with the following program: $98^{\circ} \mathrm{C}$ for $30 \mathrm{sec}, 25$ cycles $\left(98^{\circ} \mathrm{C}\right.$ for $10 \mathrm{sec}, 60^{\circ} \mathrm{C}$ for $15 \mathrm{sec}$, and $72^{\circ} \mathrm{C}$ for $90 \mathrm{sec}$ ), and $72^{\circ} \mathrm{C}$ for $7 \mathrm{~min}$. The PCR products were separated on agarose gel $(2 \%$ $\mathrm{w} / \mathrm{v}$ ) during $2 \mathrm{~h}$ at $140 \mathrm{~V}$ and stained with ethidium bromide.

\section{NUDX1 enzyme assay and kinetic parameters analysis using LC-MS}

In order to determine the kinetic parameters of NUDX1 proteins in vitro, NUDX1 genes were amplified and cloned into destination vectors pHNGWA using the Gateway ${ }^{\circledR}$ technique, where the NUDX1 protein was fused with the protein NusA (Busso et al., 2005). The final constructs were transformed into the bacterial strain Novagen ${ }^{\circledR}$ Rosetta (Merck, Darmstadt, Germany). Native proteins $(\sim 16 \mathrm{kDa})$ were obtained by using TALON ${ }^{\circledR}$ metal affinity resins (Clontech Laboratories, California, USA) to cleave away the NusA tags under the reaction of thrombin. Purified native proteins (5-56 ng) were added to substrates GPP, FPP, IPP, dGTP, or 8-oxodGTP, ranging from 0.1 to $100 \mu \mathrm{m}$. The proteins and the substrates were incubated in reaction buffer $(50 \mathrm{~mm}$ Tris- $\mathrm{HCl}$ [pH 8.8], $5 \mathrm{~mm}$ $\mathrm{MgCl}_{2}, 14 \mathrm{~mm} \beta$-mercaptoethanol, $10 \%$ glycerol, v/v) with a final volume of $100 \mu \mathrm{l}$, for $15 \mathrm{~min}$ at $30^{\circ} \mathrm{C}$ with shaking. In parallel, for each assay, a similar negative control that only contained corresponding amounts of substrates was also included. The reactions were stopped by adding $10 \mu \mathrm{l}$ of EDTA ( $100 \mathrm{~mm}, \mathrm{pH}$ 8) and $90 \mu \mathrm{l}$ of ethanol/0.5\% $\mathrm{NH}_{4} \mathrm{OH}$ solution, according to a previously published protocol (Magnard et al., 2015).

The NUDX1-dependent production of GP or FP was calculated after subtracting the GP or FP amounts present in the corresponding negative control set; $K_{M}$ and $k_{c a t}$ values were calculated by fitting the data (a series of GPP/FPP concentrations and the GP/FP conversion rates) to the Michaelis-Menten equation using $R(R$
Core Team, 2015). For substrate specificity studies and the analysis of reaction products, experiments were performed according a previously published protocol (Magnard et al., 2015) with slight adjustments as follows. Purified NUDX1 proteins (500-1000 ng) were incubated with different substrates (GPP, FPP, IPP, and 8oxo-dGTP) at a concentration of $\sim 20 \mu \mathrm{m}$ in reaction buffer (100 $\mu \mathrm{l}$ final volume) for $1 \mathrm{~h}$ at $30^{\circ} \mathrm{C}$ with shaking at $7 \times 100 \mathrm{~min}^{-1}$. The parameters and equipment settings were given in a previously published study (Magnard et al., 2015).

\section{Expression of NUDX1 in $\mathbf{N}$. benthamiana and LC-MS analysis of terpenol glycosides}

For transient expression assays, four constructs were transformed into Agrobacterium tumefaciens strain C58 (pMP90). These constructs allow, respectively, the expression of the following proteins: RwNUDX1-2c (35S:RwNUDX1-2c), RhNUDX1 (Magnard et al., 2015), GFP (35S:GFP), and the viral suppressor of gene silencing p19 (Voinnet et al., 2003). The A. tumefaciens cultures were grown overnight and diluted in infiltration buffer ( $\mathrm{pH} 5.6,2 \%$ glucose, $5 \mathrm{~g} \mathrm{~L}^{-1} \mathrm{MS}$ salt, $10 \mathrm{~mm}$ MES, and $0.2 \mathrm{~mm}$ acetosyringone) to obtain a final $\mathrm{OD}_{600}$ value of 0.6 . Before infiltration, the cultures corresponding to NUDX1 and GFP constructs were mixed with the suppressor construct culture in a ratio of 1:1. The bacterial culture mixtures were kept in the dark at room temperature for at least $1 \mathrm{~h}$ prior to infiltration. Four-week-old non-flowering $N$. benthamiana plants were used. Young leaves were infiltrated either with $A$. tumefaciens cultures harboring a NUDX1 construct (35S: RwNUDX1-2c or 35:RhNUDX1) or the 35S:GFP control, all in combination with 35S:P19. Three days after infiltration, $0.75 \pm 0.1 \mathrm{~g}$ of fresh transformed leaves were collected, immediately frozen in liquid nitrogen, and stored at $-80^{\circ} \mathrm{C}$ before use. Frozen leaves were freeze-dried prior to extraction and analysis. Terpenol glycosides were extracted from powdered freeze-dried $N$. benthamiana leaves with methanol, using $20 \mu \mathrm{l}$ of methanol per mg of dry weight. The samples were subjected to sonication for $10 \mathrm{~min}$ in an ultrasonic bath (Elma, Singen, Germany) and centrifuged (11 $292 \mathrm{~g}, 10 \mathrm{~min}, 4^{\circ} \mathrm{C}$ ). The supernatants were recovered for analysis. Terpenol glycosides were analyzed using LC-MS as described previously (Magnard et al., 2015). Terpenol glycosides were quantified using their respective characteristic ion: $\left[\mathrm{C}_{10} \mathrm{H}_{17}\right]^{+}(\mathrm{m} / \mathrm{z}$ 137.1325) for geranyl glycosides and $\left[\mathrm{C}_{15} \mathrm{H}_{24}\right]^{+}(\mathrm{m} / \mathrm{z} 205.1952)$ for farnesyl glycosides. The major geranyl glycosides were putatively identified as hexosyl-geraniol $\left(\mathrm{C}_{16} \mathrm{H}_{28} \mathrm{O}_{6}\right)$, malonyl-hexosyl-geraniol $\left(\mathrm{C}_{19} \mathrm{H}_{30} \mathrm{O}_{9}\right)$, and pentosyl-hexosyl-geraniol $\left(\mathrm{C}_{21} \mathrm{H}_{36} \mathrm{O}_{10}\right)$. Similarly, the main farnesyl glycosides were putatively identified as hexosyl-farnesol $\left(\mathrm{C}_{21} \mathrm{H}_{36} \mathrm{O}_{6}\right)$ and malonyl-hexosyl-farnesol $\left(\mathrm{C}_{24} \mathrm{H}_{38} \mathrm{O}_{9}\right)$. For each sample, total relative amounts of geranyl glycosides were obtained by summing the peak areas corresponding to the different geranyl glycosides; the same was done for farnesyl glycosides. For each construct, eight independent biological replicates were used.

\section{GC-MS analysis of rose petal extracts}

For volatile extraction, rose petals were collected between 8:30 and 10:30 in the morning. Each year, for each individual of the OW progeny, petals from different flowers on the same individual were harvested (from four to six independent flowers, depending on the number of petals per flower). The petals were mixed, and two to four technical replicates were prepared by placing $1 \mathrm{~g}$ of petals into a glass vial for volatile compound extraction. One gram of collected rose petals was incubated in $2 \mathrm{ml}$ of hexane containing $5 \mathrm{mg} \mathrm{L}^{-1}$ camphor as internal standard, at $4^{\circ} \mathrm{C}$ overnight. On the next day, the volatile extracts were transferred to a vial for GC- 
MS analysis. Extracts were analyzed on an Agilent 6850 Network GC system equipped with a DB5 apolar capillary column $(30 \mathrm{~m} \times 0.25 \mathrm{~mm})$ and coupled with a 7683B series injector and 5973 Network mass selective detector (all components from Agilent Technologies, Santa Clara, USA). The carrier gas was helium at a flow rate of $1.0 \mathrm{ml} \mathrm{min}{ }^{-1}$. The separation was performed with the following program: $40^{\circ} \mathrm{C}$ for $3 \mathrm{~min}$, and subsequently a gradient of $3^{\circ} \mathrm{C} \mathrm{min}^{-1}$ was applied until the temperature reached $245^{\circ} \mathrm{C}$. The injection volume was $2 \mu \mathrm{l}$ with a split mode (split ratio 1:2) and the injector and detector temperatures were $250^{\circ} \mathrm{C}$. The parameters for the mass spectrometer detector were set as follows: the mass scan range was $35-450 \mathrm{~m} / \mathrm{z}$ and the ionization energy was $70 \mathrm{eV}$. The identification of volatile compounds was based on their retention time and the calculated Kovats retention index in combination with their mass spectrum matching with available databases (CNRS, Wiley 275, NIST08), using both Agilent software MSD ChemStation D.02.00.275 and an archive of mass spectra of essential oil components (Adam, 2007). The farnesol produced by Rw was extracted and sent to the company International Flavors \& Fragrances (IFF LMR Naturals, Grasse, France) for isomer identification using the isomer-isolated farnesol standard. Data were analyzed by R software (R Core Team, 2015) to examine the correlations between data and determine the distribution of the compounds and the variance between replicated samples from the same genotype.

\section{RhNUDX1 purification and crystal structure determination}

A plasmid allowing the expression of RhNUDX1 protein in E. coli was constructed by inserting the RhNUDX1 gene using the Gateway ${ }^{\circledR}$ technique in the pHGWA vector (Busso et al., 2005), which adds $\mathrm{His}_{6}$ tag to the $\mathrm{C}$-terminus of the protein. RhNUDX1 protein was overproduced by transforming E. coli Rosetta 2 (DE3) competent cells by this plasmid. A single colony was used to inoculate a $5-\mathrm{ml}$ preculture in Lysogeny broth (LB) supplemented with $35 \mu \mathrm{g} \mathrm{ml}^{-1}$ chloramphenicol and $100 \mu \mathrm{g} \mathrm{ml}^{-1}$ ampicillin. The bacteria were then transferred to $1 \mathrm{~L}$ Terrific Broth supplemented with $0.8 \%$ glycerol, $35 \mu \mathrm{g} \mathrm{ml}^{-1}$ chloramphenicol, and $100 \mu \mathrm{g} \mathrm{ml}^{-1}$ ampicillin and grown at $18^{\circ} \mathrm{C}$ for $24 \mathrm{~h}$. Bacteria were harvested by centrifugation and the cell pellet was suspended in $50 \mathrm{~mm}$ Tris$\mathrm{HCl}(\mathrm{pH} \mathrm{8.0)}, 200 \mathrm{~mm} \mathrm{NaCl}, 5 \mathrm{~mm}$ dithiothreitol (DTT), and $1 \mathrm{~mm}$ phenylmethane sulfonyl fluoride (PMSF) and lysed by sonication. The lysate was applied to a Ni-NTA affinity column equilibrated in $50 \mathrm{~mm}$ Tris- $\mathrm{HCl}(\mathrm{pH} 8.0), 200 \mathrm{~mm} \mathrm{NaCl}, 5 \mathrm{~mm}$ DTT, and $20 \mathrm{~mm}$ imidazole. RhNUDX1 protein was eluted with a linear gradient from 20 to $500 \mathrm{~mm}$ of imidazole in the buffer used to equilibrate the column. Fractions containing RhNUDX1 were collected and treated with $200 \mathrm{U}$ of thrombin at $4^{\circ} \mathrm{C}$ for $18 \mathrm{~h}$ in order to cleave the $\mathrm{His}_{6}$ tag. RhNUDX1 was further purified using a Superdex 200 gel filtration column and concentrated in Amicon ${ }^{\circledR}$ (Millipore, Guyancourt, France) to $2.5 \mathrm{mg} \mathrm{ml}^{-1}$. Crystallization assays were performed on RhNUDX1 with and without its GPP substrate. Crystals were obtained in $200 \mathrm{~mm}$ ammonium sulfate, $100 \mathrm{~mm}$ sodium acetate (pH 4.6), and 25\% PEG 400. Crystals were cryoprotected in $30 \%$ ethylene glycol and flash-frozen in liquid nitrogen. GPP-containing crystals were obtained by soaking the crystals $1 \mathrm{sec}$ in $5 \mathrm{~mm}$ GPP without divalent cations before flash-freezing in liquid nitrogen. Diffraction data were collected on the synchrotron SOLEIL beamline Proxima-2 for RhNUDX1 and on the ESRF beamline ID29 for RhNUDX1/GPP. Data were integrated and scaled with DIALS (Winter et al., 2018). Phases were determined by molecular replacement in PHASER (Phaser crystallographic software) (McCoy et al., 2007) using the structure of MutT (PDB code 4KYX) as search model. Modeling and refinement were carried out using COOT (Emsley et al., 2010) and Phenix (Afonine et al., 2012).

\section{Modeling of protein structures}

Protein-ligand complexes for AtNUDX1 and RhNUDX1 were modeled using their respective crystal structures. Other Rose NUDX1 structures (RcNUDX1-1a, RcNUDX1-1b, and RwNUDX12c) were generated by homology modeling with the program MODELLER (Šali and Blundell, 1993). In order to remove bias from a single template, several structures were used as templates: the crystal structure of AtNUDX1 in complex with IPP (PDB code 6DBZ), 8-oxo-dGTP (PDB code 6FL4), and GPP (PDB code 5GP0), MutT and MTH1 in complex with 8-oxo-GMP (PDB codes 3A6T and 3ZR0, respectively), and RhNUDX1 in complex with GPP (this work, PDB code 6YPF). A structure-based alignment with the target sequence and the template structures was generated with MODELLER and manually corrected. In a first step, the ligand was added as a rigid molecule. Structures were generated with the automodel routine of MODELLER. The $\mathrm{Mg}^{2+}$ ions and ligand, when absent from the structure, were added and positioned according to AtNUDX1- $\mathrm{Mg}^{2+}$-ligand structures (6DBZ and 6FL4). Ligand topology was added in the MODELLER database. The protein-ligand complex was locally refined with MODELLER. The structures were sorted according to their Discrete Optimized Protein Energy (DOPE) score (Shen and Šali, 2006), and the structure with the lowest DOPE score was chosen as the prototype of the protein-ligand structure. In a second step, the structure of the protein-ligand complex, either GPP, FPP, or 8-oxo-dGTP, was refined by MD simulation. The coordinates and the topology of the ligands were generated with the CGenFF server (Vanommeslaeghe and MacKerell, 2012). $\mathrm{Mg}^{2+}$ ions were added in the active site, as they are important for binding and catalysis. The charge of the $\mathrm{Mg}^{2+}$ was calculated with AMBER (Case et al., 2005). The structure was then subjected to energy minimization and $20 \mathrm{~ns}$ MD with GROMACS (version 2018.3) (Abraham et al., 2015). Input parameter files for calculations with GROMACS using the CHARMM36 force field were generated with CHARMM-GUI (Lee et al., 2016). The protein-ligand complex was equilibrated in explicit solvent with $150 \mathrm{mM} \mathrm{KCl}$ in a cubic box with periodic boundary conditions at $303 \mathrm{~K}$ and 1 bar. The MD trajectory was analyzed with the tools provided with GROMACS. The protein-ligand binding energy was evaluated with PRODIGYLIG (Kurkcuoglu et al., 2018). The molecular structure figures were prepared with PyMOL (Schrödinger, 2015).

\section{ACCESSION NUMBERS}

Sequence data from this article can be found in the GenBank/European Molecular Biology Laboratory databases (see accession numbers in Table S8). Transcriptomic data are available from the European Nucleotide Archive under accession number PRJEB37774. The structures of RhNUDX1 and RhNUDX1/GPP were deposited to the protein databank under the accession codes 6YPB and 6YPF, respectively.

\section{ACKNOWLEDGEMENTS}

This work was supported by grants from the Région Rhône-Alpes, CNRS GDR MediatEC (3658), and ANR Rosascent. We thank Florence Nicolè (UJM Saint-Etienne) for helping with statistical analysis. We thank F. Hache from the English department of SaintEtienne University for text revisions. We also thank F. Gros for technical assistance (UJM Saint-Etienne). We thank Thuy-Thanh Truong (INRAE, Colmar) for help with the terpenol glycoside analysis. We thank the experiment unit Hortis (Beaucouzé, France) for 
taking care of the OW progeny. We thank the staff at the synchrotron SOLEIL beamline Proxima- 2 and at the ESRF beamline ID29 for support in data collection. We thank IFF-LMR Naturals (Grasse, France) for help with compound identification.

\section{AUTHOR CONTRIBUTIONS}

SB conceived the original screening and research plans; $\mathrm{SB}, \mathrm{RCS}, \mathrm{PH}, \mathrm{J}-\mathrm{CC}, \mathrm{CT}, \mathrm{FF}$, and $\mathrm{MH}$ supervised the experiments. PS, $C D, A B$, and $S R$ performed most of the experiments. J-LM, LH-SO, TT, JJ, JM-M, AME-H, CC, SNP, and RB performed some experiments and gene expression analyses and provided conceptual and/or technical assistance to PS, CD, and $A B$; SB, RCS, CT, SR, and PH designed the experiments; $\mathrm{SB}, \mathrm{PS}$, and $\mathrm{PH}$ wrote the article with contributions from all the authors.

\section{CONFLICT OF INTEREST}

The authors declare that there is no conflict of interest associated with the manuscript.

\section{SUPPORTING INFORMATION}

Additional Supporting Information may be found in the online version of this article.

Table S1. Sequences in RNA-seq transcriptome with homologies to NUDX1 sequences.

Table S2. List of PCR primers.

Table S3. Identity percentages of amino acid sequences among NUDX1 proteins.

Table S4. Summary of QTLs for sesquiterpenoids detected with the non-parametric Kruskal-Wallis rank-sum test.

Table S5. Summary of OTLs for sesquiterpenoids detected with interval mapping.

Table S6. Data collection and refinement statistics.

Table S7. Summary of value analysis during $20 \mathrm{~ns}$ MD.

Table S8. Accession numbers of the NUDX1 sequences.

Figure S1. Alignment of amino acid sequences of four RcNUDX11a copies.

Figure S2. Distribution in the OW progeny of volatile compound amounts.

Figure S3. Localization of the QTLs for E,E-farnesol and other sesquiterpenoid compounds.

Figure S4. Microsynteny analysis at the NUDX1-2c locus.

Figure S5. Amino acid sequence alignment and structure indication of NUDX1 proteins.

Figure S6. Analysis of the 20 ns trajectories for NUDX1 proteins.

Figure S7. Models of NUDX1-substrate interactions.

Data S1. qPCR data of 18 rose individuals and their corresponding major volatile compounds and correlation coefficient values generated by Spearman's correlation test.

Data S2. Major volatile compounds extracted from petals of 148 progeny and two parents as analyzed by GC-MS.

\section{REFERENCES}

Abraham, M.J., Murtola, T., Schulz, R., Páll, S., Smith, J.C., Hess, B. and Lindahl, E. (2015) GROMACS: high performance molecular simulations through multi-level parallelism from laptops to supercomputers. SoftwareX, 1-2, 19-25.
Adam, R.P. (2007) Identification of essential oil components by gas chromatography/mass spectrometry, 4th edition. Carol Stream, IL: Allured Publ. Corporation.

Afonine, P.V., Grosse-Kunstleve, R.W., Echols, N., Headd, J.J., Moriarty, N.W., Mustyakimov, M., Terwilliger, T.C., Urzhumtsev, A., Zwart, P.H. and Adams, P.D. (2012) Towards automated crystallographic structure refinement with phenix.refine. Acta Cryst. D, 68, 352-367.

Bellés, X., Martín, D. and Piulachs, M.-D. (2005) The mevalonate pathway and the synthesis of juvenile hormone in insects. Annu. Rev. Entomol. 50, 181-199.

Bergougnoux, V., Caissard, J.C., Jullien, F., Magnard, J.L., Scalliet, G., Cock, J.M., Hugueney, P. and Baudino, S. (2007) Both the adaxial and abaxia epidermal layers of the rose petal emit volatile scent compounds. Planta, 226, 853-866.

Bessman, M.J. (2019) A cryptic activity in the Nudix hydrolase superfamily. Protein Sci. 28, 1494-1500

Bessman, M.J., Frick, D.N. and O'Handley, S.F. (1996) The MutT Proteins or "Nudix" hydrolases, a family of versatile, widely distributed, "housecleaning" enzymes. J. Biol. Chem. 271, 25059-25062.

Bolger, A.M., Lohse, M. and Usadel, B. (2014) Trimmomatic: a flexible trimmer for Illumina sequence data. Bioinformatics, 30, 2114-2120.

Busso, D., Delagoutte-Busso, B. and Moras, D. (2005) Construction of a set Gateway-based destination vectors for high-throughput cloning and expression screening in Escherichia coli. Anal. Biochem. 343, 313-321.

Cao, L., Zhang, P. and Grant, D.F. (2009) An insect farnesyl phosphatase homologous to the $\mathrm{N}$-terminal domain of soluble epoxide hydrolase. Biochem. Biophys. Res. Commun. 380, 188-192.

Carter, M., Jemth, A.S., Hagenkort, A. et al. (2015) Crystal structure, biochemical and cellular activities demonstrate separate functions of $\mathrm{MTH}$ and MTH2. Nat. Commun. 6, 7871.

Case, D.A., Cheatham, T.E. III, Darden, T., Gohlke, H., Luo, R., Merz, K.M. Jr, Onufriev, A., Simmerling, C., Wang, B. and Woods, R.J. (2005) The Amber biomolecular simulation programs. J. Comput. Chem. 26, 16681688.

Chen, W. and Viljoen, A.M. (2010) Geraniol - a review of a commercially important fragrance material. S. Afr. J. Bot. 76, 643-651.

Chen, X., Wang, Y., Sun, J., Wang, J., Xun, H. and Tang, F. (2016) Cloning, expression and functional characterization of two sesquiterpene synthase genes from moso bamboo (Phyllostachys edulis). Protein Expr. Purif. 120, 1-6.

Cheng, A.X., Xiang, C.Y., Li, J.X., Yang, C.Q., Hu, W.L., Wang, L.J., Lou, Y.G. and Chen, X.Y. (2007) The rice (E)-beta-caryophyllene synthase (OsTPS3) accounts for the major inducible volatile sesquiterpenes. Phytochemistry, 68, 1632-1641.

Churchill, G.A. and Doerge, R.W. (1994) Empirical threshold values for quantitative trait mapping. Genetics, 138, 963-971.

De Loof, A. and Schoofs, L. (2019) Mode of action of Farnesol, the "noble unknown" in particular in $\mathrm{Ca}^{2+}$ homeostasis, and its juvenile hormoneesters in evolutionary retrospect. Front. Neurosci. 13, 141.

Dobrzanska, M., Szurmak, B., Wyslouch-Cieszynska, A. and Kraszewska, E. (2002) Cloning and characterization of the first member of the Nudix family from Arabidopsis thaliana. J. Biol. Chem. 277, 50482-50486.

Dong, L., Miettinen, K., Goedbloed, M., Verstappen, F.W.A., Voster, A. Jongsma, M.A., Memelink, J., Krol, S.V.D. and Bouwmeester, H.J. (2013) Characterization of two geraniol synthases from Valeriana officinalis and Lippia dulcis: similar activity but difference in subcellular localization. Metab. Eng. 20, 198-211.

Dubois, A., Carrere, S., Raymond, O. et al. (2012) Transcriptome database resource and gene expression atlas for the rose. BMC Genom., 13, 638648.

Emsley, P., Lohkamp, B., Scott, W.G. and Cowtan, K. (2010) Features and development of Coot. Acta Cryst. D, 66, 486-501.

Gad, H., Koolmeister, T., Jemth, A.-S. et al. (2014) MTH1 inhibition eradicates cancer by preventing sanitation of the dNTP pool. Nature, 508 215-221.

Grabherr, M.G., Haas, B.J., Yassour, M. et al. (2011) Full-length transcriptome assembly from RNA-Seq data without a reference genome. Nat. Biotechnol. 29, 644-652.

Green, S.A., Chen, X., Nieuwenhuizen, N.J., Matich, A.J., Wang, M.Y., Bunn, B.J., Yauk, Y.-K. and Atkinson, R.G. (2012) Identification, functional characterization, and regulation of the enzyme responsible for 
floral (E)-nerolidol biosynthesis in kiwifruit (Actinidia chinensis). J. Exp. Bot. 63, 1951-1967.

Gunawardana, D., Likic, V.A. and Gayler, K.R. (2009) A comprehensive bioinformatics analysis of the Nudix superfamily in Arabidopsis thaliana. Comp. Funct. Genomics, 2009, 820381.

Guterman, I., Shalit, M., Menda, N. et al. (2002) Rose scent: genomics approach to discovering novel floral fragrance-related genes. Plant Cell, $14,2325-2338$

Henry, L.K., Thomas, S.T., Widhalm, J.R., Lynch, J.H., Davis, T.C., Kessler, S.A., Bohlmann, J., Noel, J.P. and Dudareva, N. (2018) Contribution of isopentenyl phosphate to plant terpenoid metabolism. Nat. Plants, 4, 721-729.

Hibrand Saint-Oyant, L., Ruttink, T., Hamama, L. et al. (2018) A high-quality genome sequence of Rosa chinensis to elucidate ornamental traits. Nat Plants, 4, 473-484.

lijima, Y., Gang, D.R., Fridman, E., Lewinsohn, E. and Pichersky, E. (2004) Characterization of geraniol synthase from the peltate glands of sweet basil. Plant Physiol. 134, 370-379.

Ito, M. and Honda, G. (2007) Geraniol synthases from perilla and their taxonomical significance. Phytochemistry, 68, 446-453.

Jemth, A.S., Scaletti, E., Carter, M., Helleday, T. and Stenmark, P. (2019) Crystal structure and substrate specificity of the 8-oxo-dGTP hydrolase NUDT1 from Arabidopsis thaliana. Biochemistry, 58, 887-899.

Klaus, S.M.J., Wegkamp, A., Sybesma, W., Hugenholtz, J., Gregory, J.F. and Hanson, A.D. (2005) A Nudix enzyme removes pyrophosphate from dihydroneopterin triphosphate in the folate synthesis pathway of bacteria and plants. J. Biol. Chem. 280, 5274-5280.

Krissinel, E. and Henrick, K. (2007) Inference of macromolecular assemblies from crystalline state. J. Mol. Biol. 372, 774-797.

Kupke, T., Caparrós-Martín, J.A., Malquichagua Salazar, K.J. and CuliáñezMacià, F.A. (2009) Biochemical and physiological characterization of Arabidopsis thaliana AtCoAse: a Nudix CoA hydrolyzing protein that improves plant development. Physiol. Plant. 135, 365-378.

Kurkcuoglu, Z., Koukos, P.I., Citro, N. et al. (2018) Performance of HAD DOCK and a simple contact-based protein-ligand binding affinity predictor in the D3R Grand Challenge 2. J. Comput. Aided Mol. Des. 32, 175 185.

Lapczynski, A., Bhatia, S.P., Letizia, C.S. and Api, A.M. (2008) Fragrance material review on farnesol. Food Chem. Toxicol. 46, S149-S156.

Lee, J., Cheng, X., Swails, J.M. et al. (2016) CHARMM-GUI input generator for NAMD, GROMACS, AMBER, OpenMM, and CHARMM/OpenMM simulations using the CHARMM36 additive force field. J. Chem. Theory Comput. 12, 405-413.

Li, B. and Dewey, C.N. (2011) RSEM: accurate transcript quantification from RNA-Seq data with or without a reference genome. BMC Bioinformatics, $12,323$.

Liu, J., Guan, Z., Liu, H., Qi, L., Zhang, D., Zou, T. and Yin, P. (2018) Structural insights into the substrate recognition mechanism of Arabidopsis GPP-bound NUDX1 for noncanonical monoterpene biosynthesis. Mol. Plant, 11, 218-221.

Magnard, J.-L., Roccia, A., Caissard, J.-C. et al. (2015) Biosynthesis of monoterpene scent compounds in roses. Science, 349, 81-83.

Masumoto, N., Korin, M. and Ito, M. (2010) Geraniol and linalool synthases from wild species of perilla. Phytochemistry, 71, 1068-1075.

McCoy, A.J., Grosse-Kunstleve, R.W., Adams, P.D., Winn, M.D., Storoni, L.C. and Read, R.J. (2007) Phaser crystallographic software. J. Appl. Cryst. 40, 658-674.

McLennan, A. (2006) The Nudix hydrolase superfamily. Cell. Mol. Life Sci. 63, 123-143.

Mildvan, A.S., Xia, Z., Azurmendi, H.F., Saraswat, V., Legler, P.M., Massiah, M.A., Gabelli, S.B., Bianchet, M.A., Kang, L.W. and Amzel, L.M. (2005) Structures and mechanisms of Nudix hydrolases. Arch. Biochem. Biophys. 433, 129-143.

Nakamura, T., Meshitsuka, S., Kitagawa, S. et al. (2010) Structural and dynamic features of the MutT protein in the recognition of nucleotides with the mutagenic 8-oxoguanine base. J. Biol. Chem. 285, 444 452 .

Nyati, P., Nouzova, M., Rivera-Perez, C., Clifton, M.E., Mayoral, J.G. and Noriega, F.G. (2013) Farnesyl phosphatase, a Corpora allata enzyme involved in juvenile hormone biosynthesis in Aedes aegypti. PLoS One, 8, e71967.
Ogawa, T., Ueda, Y., Yoshimura, K. and Shigeoka, S. (2005) Comprehensive analysis of cytosolic Nudix hydrolases in Arabidopsis thaliana. J. Biol. Chem. 280, 25277-25283.

Ogawa, T., Yoshimura, K., Miyake, H., Ishikawa, K., Ito, D., Tanabe, N. and Shigeoka, S. (2008) Molecular characterization of organelle-type Nudix hydrolases in Arabidopsis. Plant Physiol. 148, 1412-1424.

Parveen, I., Wang, M., Zhao, J. et al. (2015) Investigating sesquiterpene biosynthesis in Ginkgo biloba: molecular cloning and functional characterization of $(E, E)$-farnesol and $\alpha$-bisabolene synthases. Plant Mol. Biol. 89, 451-462.

Pfaffl, M.W. (2001) A new mathematical model for relative quantification in real-time RT-PCR. Nucleic Acids Res. 29, e45.

R Core Team. (2015) R: A language and environment for statistical comput ing. Vienna, Austria: R Foundation for Statistical Computing

Raymond, O., Gouzy, J., Just, J. et al. (2018) The Rosa genome provides new insights into the domestication of modern roses. Nat. Genet. 50 772-777.

Robinson, M.D., McCarthy, D.J. and Smyth, G.K. (2010) edgeR: a Bioconductor package for differential expression analysis of digital gene expression data. Bioinformatics, 26, 139-140.

Robinson, M.D. and Oshlack, A. (2010) A scaling normalization method for differential expression analysis of RNA-seq data. Genome Biol. 11, R25.

Roccia, A., Hibrand-Saint Oyant, L., Cavel, E. et al. (2019) Biosynthesis of 2 phenylethanol in rose petals is linked to the expression of one allele of RhPAAS. Plant Physiol. 179, 1064-1079.

Rusdi, N.A., Goh, H.H., Sabri, S., Ramzi, A.B., Mohd Noor, N. and Baharum, S.N. (2018) Functional characterisation of new sesquiterpene synthase from the Malaysian herbal plant, Polygonum minus. Molecules, 23, 1370

Sali, A. and Blundell, T.L. (1993) Comparative protein modelling by satisfaction of spatial restraints. J. Mol. Biol. 234, 779-815.

Schnee, C., Kollner, T.G., Gershenzon, J. and Degenhardt, J. (2002) The maize gene terpene synthase 1 encodes a sesquiterpene synthase catalyzing the formation of $(E)$ - $\beta$-farnesene, $(E)$-nerolidol, and $(E, E)$-farnesol after herbivore damage. Plant Physiol. 130, 2049-2060.

Schrödinger, L.L.C. (2015) The PyMOL Molecular Graphics System, Version 1.8. https://pymol.org.

Schwab, W., Davidovich-Rikanati, R. and Lewinsohn, E. (2008) Biosynthesis of plant-derived flavor compounds. Plant J. 54, 712-732.

Setoyama, D., Ito, R., Takagi, Y. and Sekiguchi, M. (2011) Molecular actions of Escherichia coli MutT for control of spontaneous mutagenesis. Mutat Res. 707, 9-14.

Shalit, M., Shafir, S., Larkov, O. et al. (2004) Volatile compounds emitted by rose cultivars: fragrance perception by man and honeybees. Israel $\mathrm{J}$ Plant Sci. 52, 245-255.

Shen, M.Y. and Šali, A. (2006) Statistical potential for assessment and prediction of protein structures. Protein Sci. 15, 2507-2524.

Simkin, A.J., Miettinen, K., Claudel, P. et al. (2013) Characterization of the plastidial geraniol synthase from Madagascar periwinkle which initiates the monoterpenoid branch of the alkaloid pathway in internal phloem associated parenchyma. Phytochemistry, 85, 36-43.

Smulders, M.J.M., Arens, P., Bourke, P.M. et al. (2019) In the name of the rose: a roadmap for rose research in the genome era. Hortic. Res. 6, 65.

Srouji, J.R., Xu, A., Park, A., Kirsch, J.F. and Brenner, S.E. (2017) The evolution of function within the Nudix homology clan. Proteins, 85, 775811.

Sun, P., Schuurink, R.C., Caissard, J.C., Hugueney, P. and Baudino, S. (2016) My way: noncanonical biosynthesis pathways for plant volatiles. Trends Plant Sci. 21, 884-894.

Svensson, L.M., Jemth, A.-S., Desroses, M., Loseva, O., Helleday, T., Högbom, M. and Stenmark, P. (2011) Crystal structure of human MTH1 and the 8-oxo-dGMP product complex. FEBS Lett. 585, 2617-2621.

Van Ooijen, J.W. (2004) MapOTL 5, Software for the mapping of quantitative trait loci in experimental populations. Wageningen, The Netherlands: Plant Research International.

Van Ooijen, J.W. (2006) JoinMap 4, Software for the calculation of genetic linkage maps in experimental populations. Wageningen, The Netherlands: Plant Research International.

Vanommeslaeghe, K. and MacKerell, A.D. Jr (2012) Automation of the CHARMM General Force Field (CGenFF) I: bond perception and atom typing. J. Chem. Inf. Model. 52, 3144-3154. 
Voinnet, O., Rivas, S., Mestre, P. and Baulcombe, D. (2003) An enhanced transient expression system in plants based on suppression of gene silencing by the p19 protein of tomato bushy stunt virus. Plant J. 33, 949-956.

Winter, G., Waterman, D.G., Parkhurst, J.M. et al. (2018) DIALS: implementation and evaluation of a new integration package. Acta Cryst. D, 74 85-97.
Yang, T., Li, J., Wang, H. and Zeng, Y. (2005) A geraniol-synthase gene from Cinnamomum tenuipilum. Phytochemistry, 66, 285-293.

Yoshimura, K., Ogawa, T., Ueda, Y. and Shigeoka, S. (2007) AtNUDX1, an 8 oxo-7,8-dihydro-2'-deoxyguanosine $5^{\prime}$-triphosphate pyrophosphohydrolase, is responsible for eliminating oxidized nucleotides in Arabidopsis. Plant Cell Physiol. 48, 1438-1449. 